\title{
Functional Retinotopy of Monkey Visual Cortex
}

\author{
Gary Blasdel and Darlene Campbell \\ Department of Neurobiology, Harvard Medical School, Boston, Massachusetts 02115
}

The operations of primary visual cortex generate continuous representations of orientation, ocular dominance, and retinotopy that, to fit in two dimensions, organize at separate but overlapping scales (e.g., 20-500 $\mu \mathrm{m}, 200 \mu \mathrm{m}$ to $5 \mathrm{~mm}$, and 2-33 $\mathrm{mm}$ ). Where their scales overlap, these organizations interact; iso-orientation contours cross ocular dominance columns at right angles, and ocular dominance columns distort retinotopy near the V1/N2 border. To explore these interactions, we developed an optical technique for visualizing retinotopy in vivo that allows us to analyze it in relation to ocular dominance and orientation patterns. Our results show local retinotopic distortions in every region of macaque V1 that we examine, including regions far from the V1/N2 border. They also show a consistent relation between local axes of distortion and ocular dominance slabs, which they intersect at angles of $\sim 90^{\circ}$. A further correlation is provided by retinotopic maps from New World primates that show less distortion (9 vs 60\%) in two species characterized by an absence of pronounced ocular dominance columns. Retinotopic maps from these New World primates also revealed an unexpected tilt of the vertical midline representation that diverged from the V1/N2 border by an angle of $\sim 20^{\circ}$. Overall, these results suggest a general tendency for slab-based organizations to distort retinotopy by representing the same part of space more than once in adjacent slabs.

Key words: retinotopy; cortical magnification; primate visual cortex; ocular dominance columns; orientation selectivity; anisotropy
Primary visual cortex (V1) is the largest of many visual areas and (in primates) the first to receive information directly from the lateral geniculate nucleus (LGN). Accordingly, it has a lateral organization that reflects that of the retina; neighboring parts represent neighboring parts of space, but only to a degree. V1 retinotopy also reflects many disruptions, including ones caused by a vast increase in numbers of neurons and ones caused by the emergence of ocular dominance and orientation columns-organizations that pre-empt retinotopic considerations at local scales. As a consequence, the overall retinotopy of V1 (Fig. 1) appears distorted (Daniel and Whitteridge, 1961; Hubel and Wiesel, 1974b; Schwartz, 1977, 1985; Hubel and Freeman, 1977; Van Essen et al., 1984; Tootell et al., 1988), and over distances $<1-3$ $\mathrm{mm}$, it has proven difficult or impossible to find (Hubel and Wiesel, 1974b).

These consequences can be interpreted differently. For example, the coarseness of V1 retinotopy can be interpreted as a consequence of columns-specifically, from disruptions at their edges that prevent any possibility of representing space continuously over distances smaller than a hypercolumn - a distance of $\sim 1 \mathrm{~mm}$ or, in the case of ocular dominance, one right and one left eye pair (Hubel and Wiesel, 1974a,b).

Received May 31, 2000; revised Aug. 1, 2001; accepted Aug. 8, 2001.

This work was supported by National Institutes of Health Grants EY06586 and EY10862 and a grant from the Human Frontiers Foundation. We thank Alessandra Angelucci, John Assad, Jack Cowan, David Hubel, Marge Livingstone, Jenny Lund, Steve Macknik, Niall McLoughlin, Isabelle Mintz, and Lawrence Sincich for excellent comments on this manuscript and Anna Laury, Ben Salter, and Megan Keeliher for invaluable assistance with the preparation of the submitted text and figures. Macaque monkeys were provided by the New England Regional Primate Research Center, and New World primates were provided by the Centers for Disease Control (Atlanta, GA).

Correspondence should be addressed to G. Blasdel at his present address: Department of Neurosurgery, Brigham \& Women's Hospital, 221 Longwood Avenue, Boston, MA 02115. E-mail: gblasdel@hms.harvard.edu.

D. Campbell's present address: Department of Biomedical Sciences, Cornell University, Ithaca, NY, 14850.

Copyright (C) 2001 Society for Neuroscience $\quad 0270-6474 / 01 / 218286-16 \$ 15.00 / 0$
However, the coarseness of V1 retinotopy can also be explained by the large number of neurons representing each retinal ganglion cell. From the number in layer $4 \mathrm{c}$ alone, for example, it is clear that the ratio is at least 100 to one, and it is likely to get larger as new response properties emerge in other layers. What is more, the emergence of different attributes (e.g., different orientations, different eyes, etc.) for the same parts of space, represented next to one another, in flanking columns, implies a further increase in one dimension. These seem likely to generate local asymmetries that are unlikely to be captured by simple scalars. Accordingly, the cortical magnification (defined conventionally as the number of millimeters representing a degree of visual angle) measured across a set of columns seems likely to exceed the magnification measured (similarly) along them (Sakitt, 1982).

This expectation has been met to some degree. Distorted magnification has been reported several times in relation to ocular dominance columns (Hubel et al., 1974; Hubel and Wiesel, 1977; Tootell et al., 1982, 1988; Van Essen et al., 1984). However, most of these reports concern the same part of macaque V1: a narrow strip near the dorsal V1/V2 border of the operculum that is particularly accessible and convenient to study because the trajectories of ocular dominance columns are known (LeVay et al., 1975). Because of their tendency to intersect the V1/V2 border at right angles, the trajectories of columns near the border tend to follow trajectories that can be predicted from the border. Hence, any increase in magnification parallel to the border, with respect to magnification perpendicular to the border, can be taken to indicate a distortion perpendicular to ocular dominance columns. Not surprisingly, most studies have reported approximately the same ratio of 1.6:1 for magnification factors measured parallel and perpendicular to the V1/V2 border. However, conclusive proof remains elusive because none of these have shown a direct relation, by measuring magnification factors and ocular dominance columns directly, in the same tissue. In addition, there is 
the problem that this relation has proven difficult to establish elsewhere in V1 and that the observed 1.6 ratio falls short of the $2: 1$ ratio expected for a doubling of magnification perpendicular to ocular dominance columns.

To explore these issues, we developed a technique for visualizing retinotopic representations in vivo that allows us to map retinotopy in combination with ocular dominance and orientation patterns. In the macaque, we find a robust relationship between ocular dominance slabs and retinotopic distortions that supports previous findings and that extends them to a finer scale. It also extends them to other regions of primary visual cortex where this relationship had not been shown before. We find additional support for this relation in New World primates, where the same procedures show much less distortion in species known to lack pronounced organizations of ocular dominance in slabs.

\section{MATERIALS AND METHODS}

Our observations are based on differential images of orientation, ocular dominance, and retinotopy from 10 macaque monkeys (Macaca mulatta) and differential images of orientation and retinotopy from two New World Primates: one squirrel monkey (Samiri sciureus) and one owl monkey (Aotus trivirgatus). Images from three (of the 10) macaque monkeys were obtained specifically for this study and analyzed quantitatively to produce values in the accompanying tables and figures. Images from the remaining seven macaque monkeys, which were collected during the course of other studies, were examined qualitatively to verify the consistency of these results. The macaque monkeys were obtained from the New England Regional Primate Center; the squirrel and owl monkeys were obtained from the Center for Disease Control (Atlanta, GA) Preparation. Each animal was prepared according to established procedures (Blasdel, 1992a,b). After induction with a combined dose of ketamine and xylazine (10 mg/kg, i.m.; $2 \mathrm{mg} / \mathrm{kg}$, i.v.), each animal was intubated and then respirated with a 2:1 mixture of $\mathrm{N}_{2} \mathrm{O}$ and $\mathrm{O}_{2}$, at a rate of 30 strokes/min and a stroke volume adjusted to maintain end-tidal $\mathrm{CO}_{2}$ at $35 \mathrm{mmHg}$. Drugs and a maintenance solution (Isolyte; 2 $\mathrm{ml} \cdot \mathrm{kg}^{-1} \cdot \mathrm{hr}^{-1}$ ) were infused through an intravenous catheter, while a thermister-controlled waterbed maintained the animal's core temperature at $37 \pm 0.5^{\circ} \mathrm{C}$. After completing these procedures, we gradually administered thiopental $\left(0.2-2 \mathrm{ml} \cdot \mathrm{kg}^{-1} \cdot \mathrm{hr}^{-1}\right)$, supplemented with fentanyl $\left(0.3 \mu \mathrm{g} \cdot \mathrm{kg}^{-1} \cdot \mathrm{hr}^{-1}\right)$ and midazolam $\left(3.0 \mu \mathrm{g} \cdot \mathrm{kg}^{-1} \cdot \mathrm{hr}^{-1}\right)$ to maintain surgical anesthesia as the ketamine-xylazine wore off. We maintained deep anesthetic levels during all surgical procedures, at the end of which we terminated the administration of nitrous oxide and respirated each animal with oxygen and/or room air throughout the imaging phase of each experiment. Anesthesia was maintained with Pentothal given at a rate sufficient to prevent reflexes [e.g., lateral canthal, assessed at hourly intervals along with the level of neuromuscular blockade that we verified (through electrical stimulation) to remain at $\approx 50 \%]$.

After the application of Ophthetic $(0.5 \%)$ and isoptoatropine $(0.5 \%)$ to the eyes (to anesthetize the cornea and paralyze accommodation), we closed the lids until the time of physiological recording, at which time they were reopened and fitted with hard, gas-permeable contact lenses. In addition to protecting the cornea, these hard lenses allowed us to correct refractive errors by adjusting the radius of curvature to bring each eye into focus on the screen of a monitor placed 1-2 m away.

Chamber implantation. We exposed the cranium by making a midline scalp incision, and we bored a $25 \mathrm{~mm}$ hole over the operculum on one side. We then secured an internally threaded stainless steel chamber, with an "O"-ring embedded in its upper rim, with screws and acrylic cement. Once the chamber had been secured, we reflected the dura and relieved tension with 6-0 sutures. We then pressed a $50 \mathrm{~mm}$ diameter polycarbonate disk against the O-ring in the rim of the chamber, with a micromanipulator that allowed the disk to be moved laterally in two dimensions. A $15 \mathrm{~mm}$ diameter hole in the center of this disk allowed a $15 \mathrm{~mm}$ diameter stainless steel cylinder to be raised and lowered within the chamber, to bring a glass window at its lower end to arbitrary heights above the surface of exposed cortex.

Physiological recordings. A $200-\mu \mathrm{m}$-wide hole through the edge of the glass window allowed us to insert a glass-insulated platinum-iridium electrode (Wolbarsht et al., 1960) that we used to record single units from visually identified locations. The receptive fields of these units allowed us to align the eyes with each other and to align the visual fields of neurons in the exposed region of cortex with stimuli presented in the center of our stimulus monitor. After completing these procedures, we paralyzed each animal partially by administering a loading dose of vecuronium bromide, followed by a steady infusion that we adjusted to maintain a $50 \%$ neuromuscular blockade (assessed at regular intervals through electrical stimulation). In our experience this (50\% blockade) allows lateral canthal reflexes to be seen when and if present, while preventing problems associated with spontaneous eye movements, as long as adequate anesthesia is maintained. The latter was monitored continuously with respect to vital signs and reflexes, which were assessed at regular intervals.

Optical recordings. We obtained differential images of activity using established procedures (Blasdel, 1992a,b), that rely chiefly on the small reflectance changes that accompany activity in cortex (Penfield, 1933; Blasdel and Salama, 1986; Grinvald et al., 1986). Although these changes are evident in unstained cortex, we usually applied a voltage-sensitive dye $(0.1 \%$ NK2367 in saline for $1 \mathrm{hr}$, followed by a saline rinse) to amplify them (Blasdel, 1992a) and thereby decrease the time needed for averaging to $\leq 4 \mathrm{~min}$.

After the completion of staining, we selected a $9.2 \times 6.9 \mathrm{~mm}^{2}$ area of cortex that we illuminated with $720 \pm 25 \mathrm{~nm}$ light $\left(\approx 4 \mu \mathrm{W} / \mathrm{mm}^{2}\right)$ and imaged with a $100 \mathrm{~mm}$ focal length objective [numerical aperture (NA) $0.1]$ that collected the reflected light and relayed it to another $(125 \mathrm{~mm}$ focal length) lens that focused it on the imaging element of a Newvicon camera (COHU; model 5300). The RS-170 output of this camera was digitized and added or subtracted to the 16 bit frame buffer of an image processor (ITI; Q512) connected to an LSI-11/73 computer. To minimize photodynamic damage, we restricted illumination to the period of frame collection, preceded by a short preillumination interval of $0.7 \mathrm{sec}$.

Image alignment. Although our convention has been to present differentially imaged patterns at their original orientations-usually, with the bottom of each frame aligned with the dorsal V1/V2 border, the spatial representations addressed by this paper are simpler to comprehend if they can be compared with the visual field directly. Accordingly, we rotated and inverted each image to align cortical retinotopy approximately with the visual field.

Differential imaging. Our procedure relies on the balanced comparison of responses to nearly identical stimuli that differ with respect to only one variable that is modulated systematically between trials, so that produces positive and negative changes during the collection of frames that are added and subtracted later on. If nothing changes between trials (i.e., the stimuli are identical because no variable is modulated), this subtraction results in a blank image (Blasdel, 1992a). If something does change, however, and it changes systematically, becoming positive and negative in phase with the collection of positive and negative frames, the different responses it induces become apparent in the final differential image. Hence, by modulating the eye receiving input, the orientation of edges, or the positions of stimuli in visual space, it is possible to induce patterns associated with ocular dominance, orientation, or the retinotopic representation of V1.

Ocular dominance. Ocular dominance images are obtained by alternately shuttering the left and right eyes, as positive and negative frames are averaged. Even though responses to either eye occur throughout cortex, causing it to be more active and reflect less light, the changes induced through a particular eye are slightly greater in its columns. Hence, the subtraction of images acquired during left eye stimulation from images acquired during right eye stimulation removes common mode elements of the response (to either eye) while inverting responses to the left (subtracted) eye, causing them to appear light. As a consequence, regions dominated by the right and left eyes appear dark and light (Fig. 2a).

Orientation selectivity. When the modulated variable concerns the orientation of aligned edges, patterns associated with orientation selectivity are derived instead. By adding positive and negative images of cortex responding (alternately) to vertical and horizontal contours, for example, one obtains an image associated with orientation selectivity, where regions more responsive to vertical and horizontal appear dark and light (Fig. 2b).

Retinotopic position. We obtained differential images of retinotopic position by using apertures to restrict stimuli to complementary, stripelike compartments during alternate trials. Because the resulting images are exquisitely sensitive to retinotopic location (see Results) and pronounced interference patterns that can result from slight misalignments of the two eyes (e.g., diplopia), we found it necessary to minimize problems associated with independent eye movements by presenting 


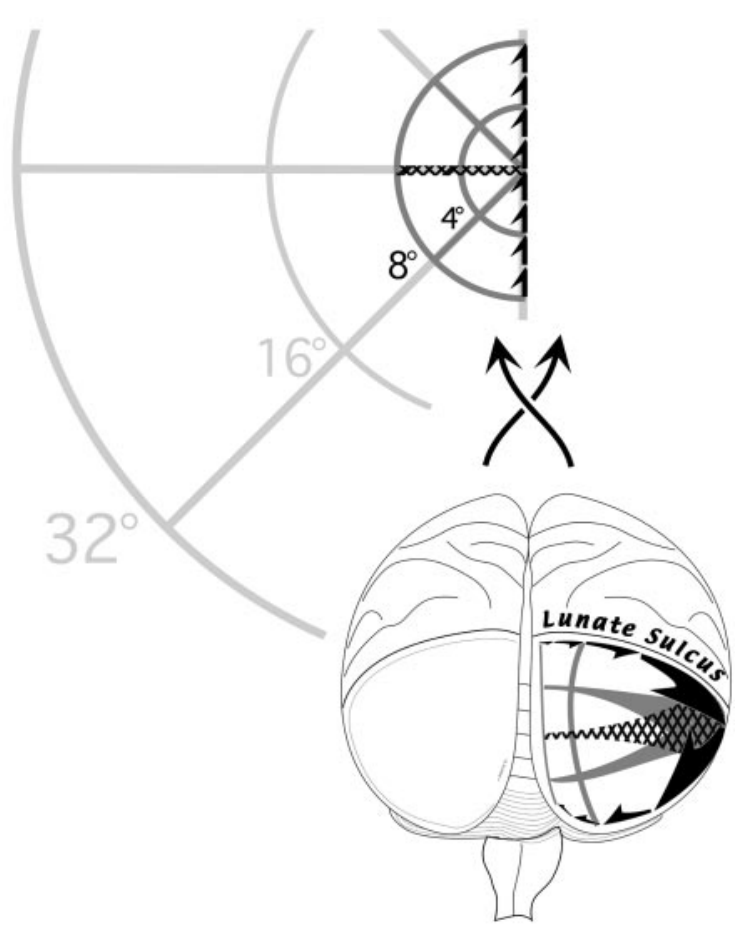

Figure 1. Representation of the contralateral visual hemifield in area V1 of the macaque monkey. The primary visual cortex (also known as striate cortex, area 17, and V1) lies at the back of the head, where it occupies $\sim 1200 \mathrm{~mm}^{2}$, an area half the size of a credit card. It is divided between two hemispheres, with each side representing the contralateral half of visual space. Approximately half the cortex on each side forms a large flat area on the operculum, under the cranium, which represents the central $8^{\circ}$ of the contralateral visual field. This diagram shows representations of vertical, horizontal, and oblique meridia on this surface to an eccentricity of $\sim 8^{\circ}$. The vertical meridian, indicated by the black half-arrows, runs along the outer boundary of V1. Because of an exponential change in magnification with eccentricity, it bulges out, encircling most of V1, and causing representations of many straight lines to appear curved and curved lines to appear straight (Bressloff et al., 2001). It is important to note that the surface representation of space is inverted around the horizontal axis. Hence, left-right relations are preserved while upper and lower parts of visual space are represented in the lower (posterior) and upper (anterior) parts of V1.

stimuli to only one eye at a time. This prevented any lasting effects from the residual, minor movement of one eye (other than a momentary reduction in contrast) that might have produced interference affecting the apparent spacing and/or trajectory of imaged bands.

Cortical neurons were activated by a standard pattern (consisting offour superimposed, nonharmonic, square wave gratings) that we presented alternately through each of two complementary sets of apertures. For the images in Figure 2, $c$ and $d$, as well as most of the images in this paper (all except those in Figs. 6 and 7), these apertures consisted of $0.7^{\circ}$ wide openings separated by $0.7^{\circ}$ masks, repeating at $1.4^{\circ}$ intervals, with the apertures and masks of the complementary set offset by $0.7^{\circ}$ to coincide with the masks and apertures of the primary set. In other words, the complementary set was offset by a $180^{\circ}$ shift in phase. By differentially imaging responses to stimuli viewed alternately through each set of apertures, we obtained patterns of dark and light bands, whose positions and orientations reflect those of primary and complementary apertures used to make them. Because Figure $2 c$ was obtained with the apertures oriented vertically (parallel to the vertical midline), for example, the induced bands run parallel to the V1/V2 border, which represents the vertical midline in the visual field. The bands in Figure $2 d$, which were induced by horizontal apertures, run perpendicular to the V1/V2 border and the bands in Figure $2 c$, although they were obtained from the same region of cortex at approximately the same time.

It is important to stress that although there may be a host of optical changes that occur in cortex in response to any stimulus and that although some of these may be quite large, they tend to be the same if care is taken to minimize the variation of extraneous variables during successive presentations. Hence, they tend to form a common mode response that is removed easily through subtraction, by the very process that reinforces modulated signals. Because the only things that change systematically during the acquisition of positive and negative frames are variables associated with: (1) the eye receiving input, (2) the orientation of edges, and/or (3) the positions of stimuli in the visual field, the resulting patterns are specific to organizations associated: (1) ocular dominance, (2) orientation selectivity, and/or (3) retinotopic location in visual space.

Signal and noise. As described previously (Blasdel, 1992a), these signals arise from activity associated with reflectance changes that usually are $<0.1 \%$. Although this lies at the threshold of changes that can be detected by video, further improvements are needed. These are achieved through averaging, by summing 1800 frames (in 300 frame increments) for each stimulus condition as well as by binning information from $4 \times 4$ squares, thereby increasing local sample sizes 16 times, at a reciprocal cost in resolution (which decreases from $512 \times 480$ to $128 \times 120$ ). When this is done, and other noise sources (e.g., blood vessels) are sufficiently avoided or suppressed, the centers of light and dark bands appear with signal-to-noise ratios of $\sim 6: 1$. These can be improved further by averaging more frames, with signal-to-noise increasing at the approximate rate of $(\sqrt{ } N) / 2$, or by filtering with a bandpass filter to remove components outside of the desired range.

Postprocessing. Because video images were collected at an initial resolution of $512 \times 480$, each pixel subtends $\approx 16$ and $12 \mu \mathrm{m}$ along horizontal and vertical axes (note that the horizontal and vertical spacing of video camera pixels differ). At the conclusion of each trial, pixels were grouped into $4 \times 4$ squares, which were then averaged and stored on disk in $128 \times 120$ arrays, from which they were resurrected and converted back to higher resolutions of $680 \times 480$ square pixels for further processing. To remove unwanted noise components at very low spatial frequencies, the latter were isolated by convolving with a Gaussian (kernel of half-width $\sigma=1.5 \mathrm{~mm}$ for ocular dominance and orientation images; $\sigma \geq 6 \mathrm{~mm}$ for retinotopy images), an approach that preserves the relative amplitudes of signals with spatial periods between 200 and 2.0 $\mathrm{mm}(\sigma=1.5)$ and between $200 \mu \mathrm{m}$ and $6 \mathrm{~mm}(\sigma \geq 6 \mathrm{~mm})$, respectively.

Contour plots. Our attempts at quantifying the spacing and trajectories of induced spatial patterns were facilitated by grids derived from contour plots, as illustrated in Figure 8. These were achieved by first removing artifacts that might cause local perturbations [i.e., by filling in from surrounding regions (see Fig. $8 a$ ), followed by convolution with a difference of Gaussians to attenuate frequencies that (for each group of images) lay outside the desired range (see Fig. 8b)]. Hence, for a collection of images whose smallest and largest apparent intervals were 2 and $5 \mathrm{~mm}$, we would have applied Gaussian kernels of half-width $\sigma \leq$ 0.25 and $\sigma \geq 2.5 \mathrm{~mm}$ symmetrically and uniformly to all patterns. Contour plots were then extracted from ridges induced by binning (see Fig. $8 c$ ) and used in combination with minima and maxima to estimate the centers of dark and light band along with contours derived from zero crossings to estimate their edges (see Fig. $8 d$ ). As one can see in the middle and lower images of Figure 9, in which the grids achieved by combining these lines appear superimposed over the patterns used to produce them, the agreement is excellent. Although final lines are drawn by eye, it is important to stress that errors in the placement of any particular line come at the expense of neighbors, whose reciprocal errors tend to cancel quickly, within 1-2 iterations (i.e., one quarter to one half cycle).

Calibration and verification of aperture spacing. Because our differentially imaged bands reflect the visual angles of apertures in space, we took care to verify the widths and intervals of the latter from the animal's perspective. We did this by monitoring their appearance with a miniature CCD camera, fitted with a normal perspective $25 \mathrm{~mm}$ lens (Zeiss, Oberkochen, Germany), which we placed at the location occupied by the animal's head during each experiment. Because of the precise geometry of the image element of the CCD, images obtained in this manner made it possible to capture and correct all sources of error in the spacing of apertures at different orientations.

Calibration of television aspect ratio and linearity. To verify the symmetry and linearity of the Newvicon camera we used to acquire images (which does not rely on a rigid, silicon-based imaging element), we photographed a $1 \times 1 \mathrm{~cm}$ grid of lines spaced at $1 \mathrm{~mm}$ intervals before and/or after each experiment to verify the uniformity of images acquired and to correct for any changes in aspect ratio over time. One additional 


\section{a}

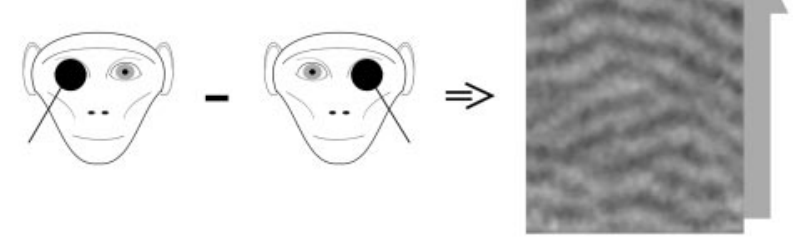

b

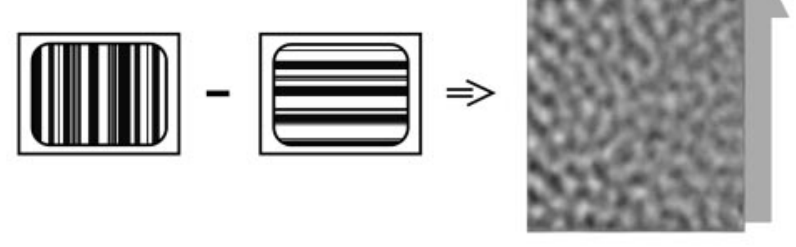

C

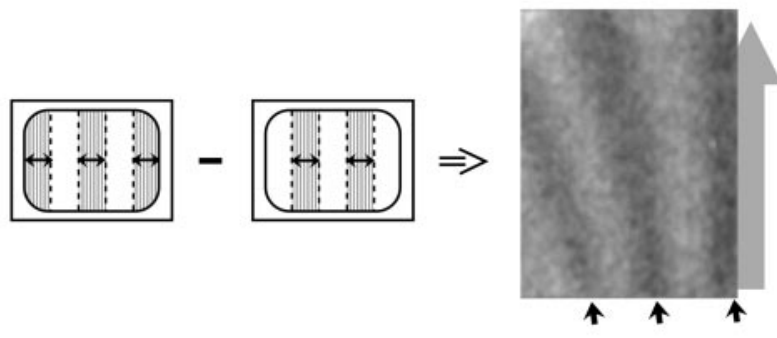

d

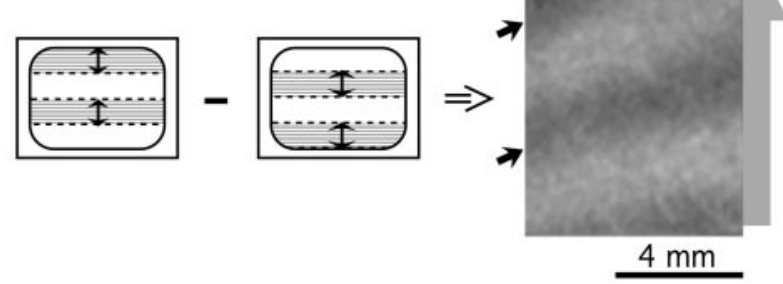

Figure 2. Differential imaging of ocular dominance, orientation, and retinotopy in monkey visual cortex. All these techniques rely on small changes in reflectance with activity, which were first observed by Penfield (1933), and used by Blasdel and Salama (1986) to visualize patterns of ocular dominance and orientation preference in vivo. Subsequent techniques have used the same or similar signals to produce similar patterns (Ts'o et al., 1990; Bonhoeffer and Grinvald, 1993). Here, we compare cortical images obtained during comparable states of activity, while one stimulus variable was modulated with the others held constant. Because all reflectance changes associated with unmodulated variables are the same, they are removed by subtraction, whereas changes associated with the modulated variable are reinforced. These principles are illustrated by four examples in $a-d$. To facilitate visual field comparisons, each of the resulting images has been rotated and inverted to bring retinotopic coordinates into alignment with visual space. To keep track of these changes, we have added a gray half-arrow to the right of each frame to indicate the side and the axis of the V1/V2 border (which represents the vertical midline) directed toward the fovea, in the direction of increasing magnification. $a$, Ocular dominance pattern. In this example, an image of ocular dominance bands was obtained by modulating the eye receiving input. One eye was covered on alternate trials. Images of cortex responding to the right eye (left eye covered) were added to the final image while images of cortex responding to the left eye were subtracted. Hence, regions responding better to the right and left eyes appear dark and light, producing a pattern of bands that projects perpendicularly into the V1/V2 border. As noted above, all the stimuli were similar in regard to basic structure and speed of movement. $b$, Pattern of orientation selectivity. The same stimulus was seen by both eyes while the orientation of edges was modulated. Images of the cortex responding to horizontal contours control that we performed entailed a $90^{\circ}$ rotation of the camera between trials (so that errors associated with aspect ratio operate in reverse) to verify that discrepancies in aspect ratio never exceeded $1 \%$.

Artifact suppression and attenuation. Because small artifacts can affect the positions of interpolated contours, we adopted a number of procedures for minimizing their impact. These entailed: (1) verifying the depth of anesthesia at frequent $(30 \mathrm{sec})$ intervals, (2) application of vasoactive drugs (e.g., atropine, propranolol, etc.), (3) repeated acquisitions of the same differential image $(>3$ for most of the images in this paper, $>6$ on some occasions), so that images that appeared particularly free of artifacts could be used. When artifacts proved unavoidable, however, we suppressed them before extracting contours by zeroing pixel values in regions that corresponded to major blood vessels and filling in the vacated regions with values extrapolated from either side. Filled-in regions never exceeded $300 \mu \mathrm{m}$ in width and never led to conclusions that contradicted those obtained from adjacent regions.

\section{RESULTS}

Figures 3 and 4 show images from two animals that we obtained from approximately the same part of primary visual cortex (V1). For each of these figures, the first four frames show: an image of the overlying vasculature $(a)$, a differential image of ocular dominance $(b)$, a differential image of $0^{\circ} / 90^{\circ}$ orientation (i.e., obtained by comparing responses with vertical and horizontal) $(c)$, and a differential image of $45^{\circ} / 135^{\circ}$ orientation $(d)$, as described extensively in previous publications (Blasdel and Salama, 1986; Blasdel, 1992a,b) along with their interactions.

Each of the four images on the right shows a differential image of position that we obtained from the same region of cortex in one animal by comparing responses to stimuli at complementary locations in the visual field. As for most of the retinotopic images in this paper, the apertures used to constrain the location of stimuli consisted of $0.7^{\circ}$ wide slits separated by $0.7^{\circ}$ wide masks, which we presented in complementary sets $\left(180^{\circ}\right.$ out of phase) at one of four orientations (vertical, left oblique, horizontal, and right oblique) in visual space. By subtracting images of cortex responding to the complementary set from images of it responding to the primary set, we obtained the patterns of dark and light bands that appear in Figure $3 e-h$. Because each of these was obtained from the same region cortex, within a relatively short time $(<30 \mathrm{~min})$, the main difference between them arises from the orientation of apertures, reflected in the orientation and the spacing of dark and light bands. Similar images from a different animal appear in Figure 4.

Because the bands in Figures $3 e$ and $4 e$ were induced by apertures that were vertical, they run parallel to the V1/V2 border (located to the right of each frame). Similarly, the bands in

$\leftarrow$

were subtracted from images of it responding to vertical contours. Hence, dark and light regions indicate preferences for vertical and horizontal. Note how different this pattern looks from that in $a$, even though both were obtained from the same region of cortex within a few hours. $c$, Retinotopic representation of vertical apertures. These patterns were obtained by modulating the position of vertically oriented stimuli seen by one eye. With the left eye covered, the same stimuli used to induce patterns in $a$ and $b$ were presented through complimentary, slit-shaped apertures on alternate trials. Hence, the regions of space containing stimuli during one trial remained blank during the other. The regions of cortex representing one set of apertures and its complement thus appear dark and light. Because the apertures used were oriented vertically, the bands run parallel to the V1/V2 border. $d$, Retinotopic representation of horizontal apertures. The paradigm used to induce this pattern was similar to that in $c$. However, in this case the apertures were oriented horizontally. Hence, the induced bands run perpendicular to the bands in $c$ as well as to the V1/V2 border. Note the wider interval for bands in this image, although the apertures used to induce them repeated at the same interval as those in $c$. 


\section{a}

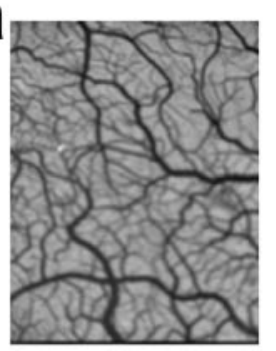

b

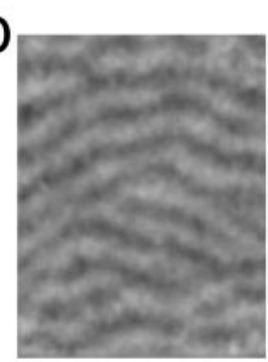

$\mathrm{C}$

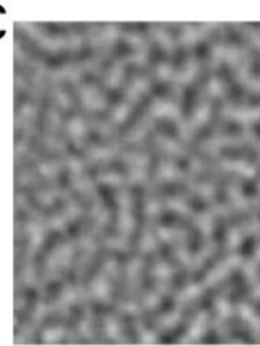

d

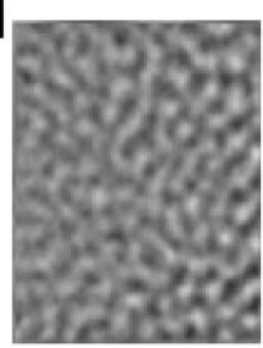

e
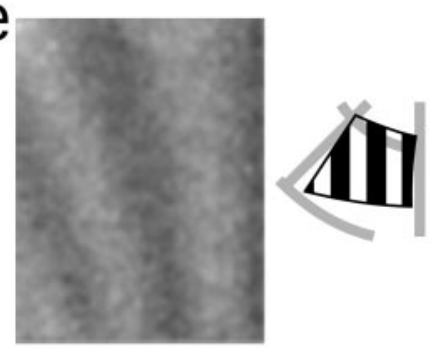

$\mathrm{f}$

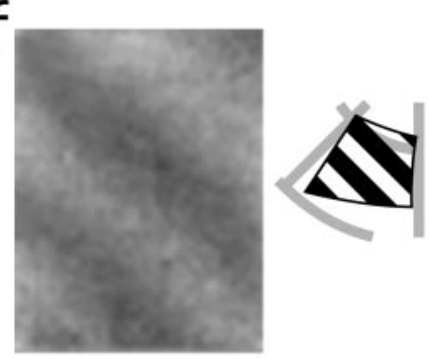

9

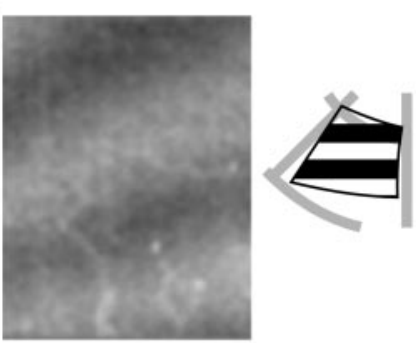

$\mathrm{h}$

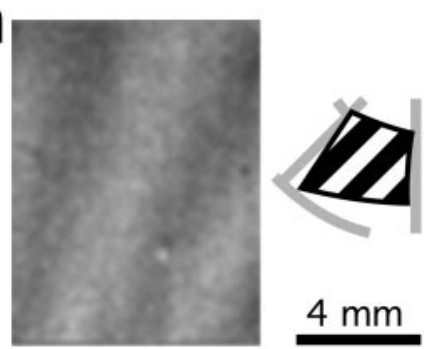

Figure 3. Differential images of ocular dominance, orientation, and four axes of retinotopic meridia, from a single region of macaque striate cortex. With the exception of $a$, which shows the vasculature, each frame shows a differential image from the same region of macaque striate cortex that we acquired with the same equipment and stimuli and within a relatively short period of time. Because we used the same stimuli on each occasion, the difference between successive patterns was the variable that was modulated. $a$, Image of the overlying vasculature, as it appeared under green $(540 \mathrm{~nm})$ light. Because light passes through each blood vessel twice (on its way in and then again on its way back out), even minor perfusion changes can lead to spurious changes in the final pattern if they are large enough, or if they correlate, even partially, with the modulated variable (Blasdel, 1992). They are included here for reference so that important conclusions can be restricted to regions where vascular artifacts are least likely (Obermayer and Blasdel, 1993). Because the $720 \mathrm{~nm}$ light we use is minimally absorbed by hemoglobin (McLoughlin and Blasdel, 1998), there are few signs of artifact in most of the imaged patterns $(b-h)$. $b$, Differential image of ocular dominance, obtained by subtracting images of cortex responding to the left eye from images of it responding to the right eye. The light and dark bands correspond to regions dominated by the left and right eyes, respectively. They run horizontally at intervals of $\sim 500 \mu \mathrm{m}$ (for each right plus left eye pair) and intersect the V1/V2 border at angles of $\sim 90^{\circ}$ (LeVay et al., 1975). $c$, Differential image of orientation selectivity for vertical and horizontal stimuli. This image was obtained by comparing responses with different orientations, rather than different eyes, and thus differs from that in $b$. Because we compare responses to vertical and horizontal stimuli, dark and light regions indicate selectivities
Figures $3 g$ and $4 g$, which were induced by horizontal apertures, project horizontally through each image to intersect the V1/V2 border (and the bands in Figs. $3 e$ and $4 e$ ) at angles of $90^{\circ}$. Also, the bands in Figures 3, $f$ and $h$, and $4, f$ and $h$, which were induced by oblique apertures, follow intermediate trajectories that intersect the V1/V2 border at angles of approximately $\pm 45^{\circ}$.

\section{Retinotopic correspondence}

As one can see, the bands in each image bear a striking resemblance to the apertures used to make them. They reflect their orientation and their spacing, but only to a degree because they also look fuzzier and less regular. The bands in Figures $3 g$ and $4 g$, for example, appear farther apart than those in Figures $3 e$ and $4 e$, although the apertures used to make them were spaced the same, and most of the bands in these images (particularly those in Figs. $3 e$ and $4 e$ ) appear to expand upward and bend toward the left, reflecting a superimposed magnification gradient that increases toward the foveal representation at the top right.

Clearly, the bands in each image reflect more than the apertures used to make them; they also carry elements of transforms in between-from the visual field to the retina, to the LGN, to their representation in (the top layers of) V1. Accordingly, it should be possible to characterize these rearrangements by relating each pattern to the stimuli used to make it, in visual space. To clarify the sensitivity and resolution of this procedure, we explored the consequences of minor perturbations of stimulus apertures by modulating: (1) their displacement (Fig. 5), (2) their intervals (Fig. 6), and (3) their widths while keeping their intervals the same (Fig. 7).

Figure 5, $a$ and $b$, explores the displacement sensitivity of induced patterns by comparing the positions of bands induced by the same stimulus apertures before and after an imposed elevation shift of $0.25^{\circ}$. Because the aperture pattern is periodic, this corresponds to $17.9 \%$ of its period $\left(1.4^{\circ}\right)$ or a $64^{\circ}$ shift in phase. As one can see, the bands in Figure $5 b$ appear higher than those in Figure $5 a$, reflecting a displacement that is especially apparent from the superimposed profiles (extracted from the indicated rectangles) in Figure $5 c$. From the relative displacement of zero-

$\leftarrow$

for vertical and horizontal. $d$, Differential image of orientation selectivity for left and right oblique stimuli. In this image obtained by subtracting right oblique responses from left oblique ones, the dark and light regions indicate selectivities for left and right oblique. Although the resulting pattern resembles the preceding one in $c$, it differs by one quarter of a cycle because of the quarter cycle $\left(180 / 4=45^{\circ}\right)$ shift in the orientations compared. Hence, the dark and light peaks in this image line up with the zero-crossings, regions showing no preference for vertical or horizontal, in $c$. Collectively, this image and that in $c$ reflect the orientation preferences and selectivities for the entire region. $e$, Differentially imaged responses to vertical $0.7^{\circ}$ apertures repeating at $1.4^{\circ}$ intervals. In this experiment, we restricted the location of stimuli to narrow strips of space, which alternated with other, complimentary strips on alternate trials. In this example, the apertures are oriented vertically. Hence, the dark and light bands, which represent each set of apertures, run parallel to the representation of the vertical meridian, itself parallel to the V1/V2 border. $f$, Differential image of bands induced by $0.7^{\circ}$ apertures, repeating at $1.4^{\circ}$ intervals, oriented at $45^{\circ}$ (left oblique). $g$, Differential image of bands induced by $0.7^{\circ}$ apertures, repeating at $1.4^{\circ}$ intervals, oriented at $90^{\circ}$ (horizontal). In this experiment, the bands run at right angles to those in $e$ and the V1/V2 border, although both were obtained from the same region of cortex within an hour of time. Also, note the wider intervals between these bands, as opposed to those in $e$, on account of a greater cortical magnification in the vertical direction (by $\sim 60 \%$ ). $h$, Differential image of bands induced by $0.7^{\circ}$ apertures, repeating at $1.4^{\circ}$ intervals, oriented at $135^{\circ}$ (right oblique). 


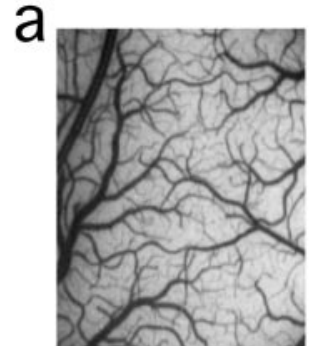

e

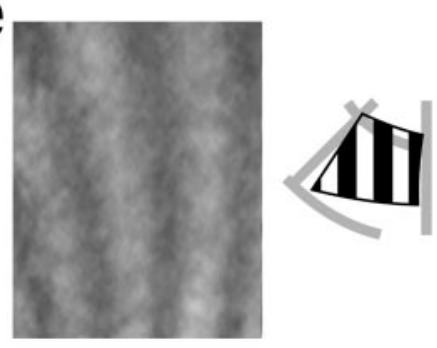

b

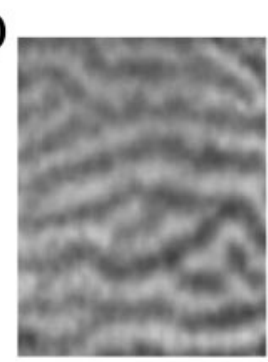

C

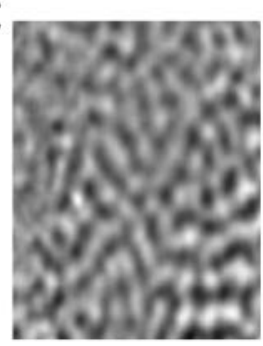

d

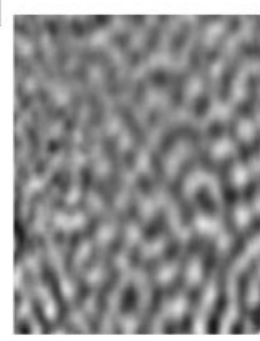

f
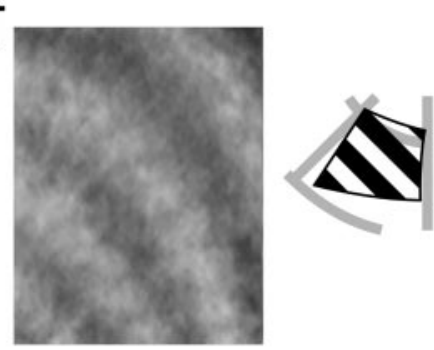

9

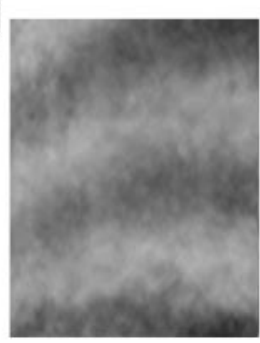

$\mathrm{h}$

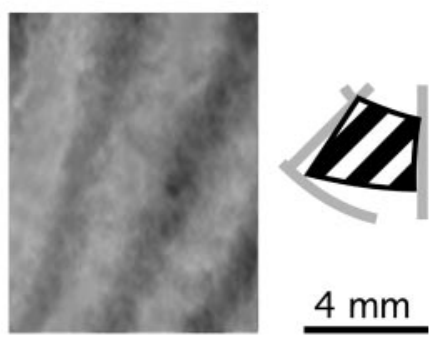

Figure 4. Differential images of ocular dominance, orientation, and four axes of retinotopic meridia, from a single part of striate cortex in another macaque monkey. These images $(a-h)$ are similar to those in Figure 3, except that they were obtained in a different animal and from a region of cortex that was located slightly posterior (with respect to the V1/V2 border) to that in Figure 3. Note the dramatically curved bands in $f$ and $g$, both of which represent straight apertures in visual space.

crossings (indicated by vertical dashed lines, drawn midway between adjacent peaks), it is clear that the imposed $0.25^{\circ}$ shift in elevation has produced a $0.74 \mathrm{~mm}$ displacement-a distance corresponding to $17.5 \%$ of the $4.17 \mathrm{~mm}$ interband interval, or a $63^{\circ}$ shift in phase. From the clear separation of well defined zero-crossings, it is clear that these patterns are remarkably sensitive to position. Accordingly, it should be possible to detect even smaller displacements with ease.

Figure 6 shows four patterns induced by two orientations of aperture, horizontal (top) and vertical (bottom), each presented at two intervals. Images on the left were induced by apertures that repeated at $1.4^{\circ}$ intervals; images on the right were induced by apertures that repeated at $0.7^{\circ}$ intervals. As one can see, the bands in each image bear a striking resemblance to the apertures used to induce them. Those in the top and bottom images follow a

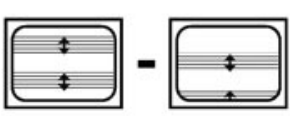

b
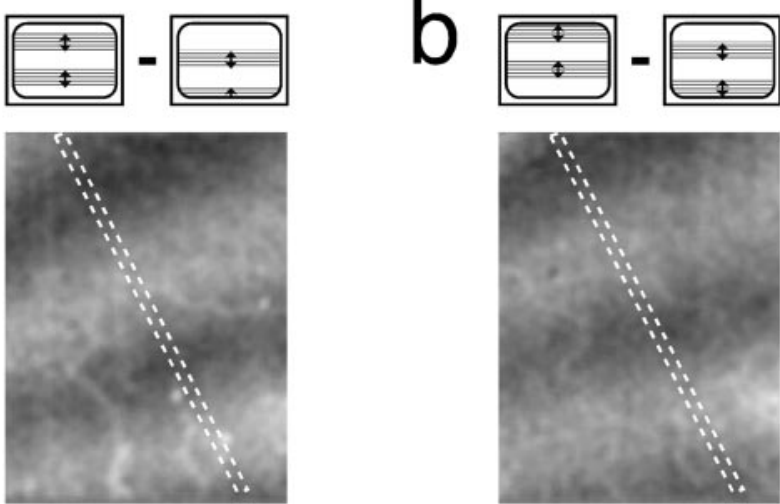

$2 \mathrm{~mm}$

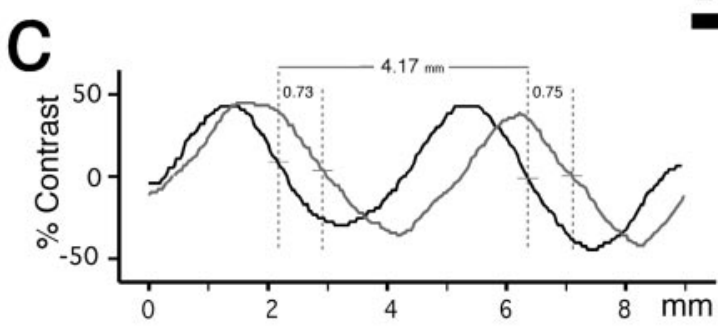

Figure 5. Sensitivity of retinotopic maps to aperture displacement. Whereas differential images of ocular dominance and orientation appear relatively insensitive to the exact location of stimuli used to make them, differential images of position are excruciatingly sensitive, as illustrated in this figure by the relative positions of patterns induced by the same apertures immediately before $(a)$ and after $(b)$ a small $\left(0.25^{\circ}\right)$ shift in gaze elevation. Because the apertures appeared at $1.4^{\circ}$ intervals, this corresponded to $17.9 \%$ of their period, or a $64^{\circ}$ shift in phase. As one can see, this produced a corresponding shift in phase that is most apparent from their profiles (obtained by graphing the average intensity along the same 0.5 -mm-wide strip, indicated by broken white lines), which appear superimposed in $c$. For each profile, the zero-crossings (indicated by vertical dashed lines) are drawn midway between positive and negative peaks on each side. As one can see, the $0.25^{\circ}$ shift produced 0.73 and 0.75 lateral displacements in the positions of zero crossings, which, considering the band period of $4.17 \mathrm{~mm}$, correspond to 17.5 and $18 \%$.

trajectories corresponding to horizontal and vertical in cortical coordinates. Those on the right appear at half the interval, and twice as numerous, as those on the left. But they also appear with much less contrast, often to the point where they barely can be seen. This is especially apparent from the cross-sectional profiles illustrated below each image. These make it clear that the bands in Figure $6 b$ appear with $67 \%$ less contrast than those in Figure $6 a$ and that the bands in Figure $6 d$ appear with $75 \%$ less contrast than those in Figure $6 c$. So, a $50 \%$ reduction in the interval between apertures clearly has reduced contrast by $>50 \%$.

Although this seems likely to reflect some interaction between cortical receptive fields and the intervals between apertures, it seems possible that it could reflect a difference in aperture width, particularly if the larger areas of stimulation allowed by wider apertures lead to greater levels of activation. To dissect these possibilities, we compared the patterns induced by apertures with different widths $\left(0.35^{\circ}\right.$ and $\left.0.7^{\circ}\right)$ but the same interval of $1.4^{\circ}$. From the resulting images in Figure 7, $a$ and $b$, it is clear that aperture width has little or no effect on peak to peak contrast as long as intervals stay the same. From the superimposed profiles in Figure $7 c$ (taken from the indicated parts of Fig. 7a,b), for example, it is clear that $0.35^{\circ}$ and $0.7^{\circ}$ wide apertures generate approximately the same sized peaks. Despite minor differences 


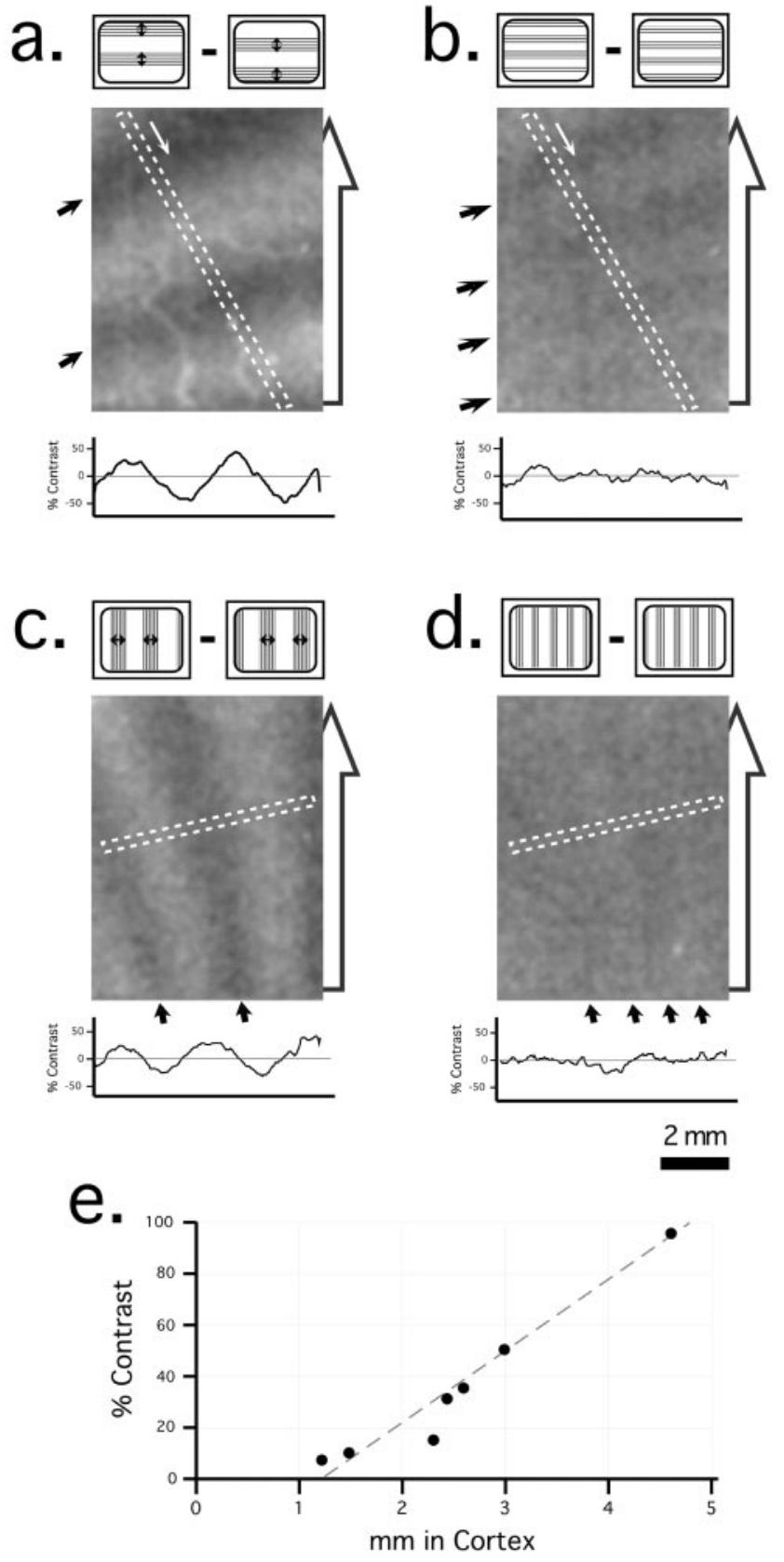

Figure 6. Relation between the contrast and separation of adjacent bands in V1. $a-d$, These four images were obtained from the same region of primary visual cortex with apertures presented at each of two orientations (top and bottom rows) and two intervals (left and right). The orientation and spacing of the induced band patterns follow these apertures closely. There is a relation between interval and contrast that reflects varying degrees of overlap between the populations activated by complementary apertures. For aperture intervals that are small enough to allow stimuli revealed by complementary apertures to activate the same receptive fields, the differences responsible for generating light and dark bands rapidly become negligible. As a consequence, smaller aperture intervals generate bands with less contrast. This raises the question of whether the observed reduction in contrast arises directly from the proximity of successively presented apertures in the visual field or from the proximity of cortical regions that become active in response. One can dissect these issues by exploiting the local magnification anisotropy (see below) in cortex to induce different band periods, along different axes in cortex, with the same aperture interval. For example, the bands in $b$ and $d$ were both induced by aperture intervals of $0.7^{\circ}$, yet the bands in $d$ lie closer than the bands in $b$, and they also appear fainter, to the point at which they barely can be seen. Accordingly, the observed effect on

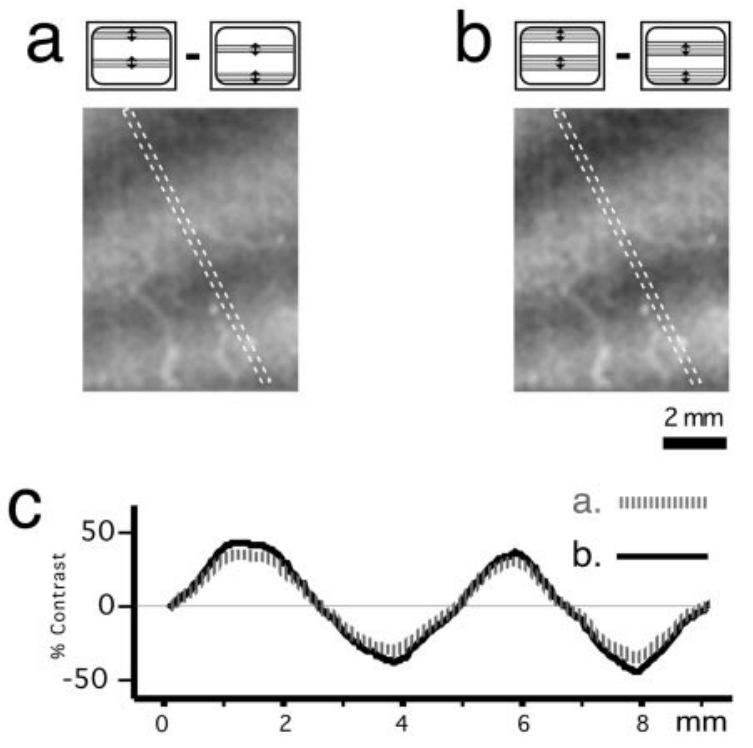

Figure 7. Minimal influence of aperture width on contrast. The images in $a$ and $b$ were both induced by horizontal apertures, repeating at $1.4^{\circ}$ intervals, but those in $a$ were induced by apertures that were only half as wide yet in phase. The intensity profile of each pattern (measured along the white lines) is represented in $c$.

between the shapes of the induced profiles, there is little to indicate a twofold difference between the areas in which stimuli were applied. Hence, the pronounced attenuations in Figure $6, b$ and $d$, would appear to reflect the intervals rather than the widths of the apertures used.

Because our technique works by comparing responses to stimuli at slightly different but complementary locations, the pronounced sensitivity to aperture interval suggests an interaction with cortical receptive fields and/or with the resolution of retinotopic representations in $\mathrm{V} 1$. To explore these interactions, we

\section{$\leftarrow$}

contrast seems to develop from the proximity of cortical activity rather than the proximity of stimulation in the visual field. A similar difference characterizes the patterns in $a$ and $c$. Although the bands in both patterns were induced by $1.4^{\circ}$ intervals, those in $c$ lie closer than those in $a$, and, the contrast between adjacent light and dark bands in $c$ appears to be less as a consequence. $a$, Horizontal apertures, repeating at $1.4^{\circ}$ intervals, induce horizontal bands that intersect the $\mathrm{V} 1 / \mathrm{V} 2$ border at right angles. $b$, Horizontal apertures at $0.7^{\circ}$ interval also induce horizontal bands that intersect the V1/V2 border at right angles. However, in this case, halving the aperture interval has doubled the number of bands (indicated by arrows). Note how this has also impaired the contrast between adjacent light and dark bands by $>50 \%$.c, Vertical apertures at $1.4^{\circ}$ intervals induce vertical bands that run parallel to the V1/V2 border. $d$, Vertical apertures at $0.7^{\circ}$ intervals induce twice as many vertical bands at vertical trajectories (see arrows), but the contrast has been reduced so much (more than that in $c$ ) that they barely can be seen. $e$, Relationship between contrast and distance. Each value represents contrast, as a function of distance, between the centers of adjacent dark and light bands. These values were obtained from six differential images of the same region of cortex (which included those in $a-d$ ) by measuring the difference between the average pixel intensities of two 100- $\mu$ m-diameter disks, located at the centers of adjacent dark and light bands, dividing by their average, and plotting their measured contrast (as a fraction of the largest value) as a function of the distance between them. Although these measurements could be made more rigorously, they illustrate a trend that is qualitatively obvious from the images in $a-d$, that interband contrast decreases linearly with distance to the indicated zero-intercept at $1.2 \mathrm{~mm}$, where it disappears. Over distances smaller than this, there is not even a suggestion of retinotopy, as one can verify qualitatively from the vanishingly faint contrast of bands in $d$. 


\begin{tabular}{|c|c|c|c|c|c|c|}
\hline & $N$ & $\begin{array}{l}\text { Aperture interval } \\
\text { (degree) }\end{array}$ & $\begin{array}{l}\text { Band interval } \\
(\mathrm{mm})\end{array}$ & $\begin{array}{l}\text { Magnification } \\
(\mathrm{mm} / \text { degree })\end{array}$ & $\mathrm{V} / \mathrm{H}$ & $\mathrm{L} / \mathrm{R}$ \\
\hline Macaque 1 & 63 & & & & & \\
\hline $\mathrm{V}_{90^{\circ}}$ & & 1.44 & 4.41 & 3.06 & 1.44 & \\
\hline $\mathrm{H}_{0^{\circ}}$ & & 1.44 & 3.07 & 2.13 & & \\
\hline $\mathrm{L}_{135^{\circ}}$ & & 1.44 & 3.62 & 2.51 & & 1.08 \\
\hline $\mathrm{R}_{45^{\circ}}$ & & 1.44 & 3.34 & 2.32 & & \\
\hline Macaque 2 & 52 & & & & & \\
\hline $\mathrm{V}_{90^{\circ}}$ & & 1.15 & 1.62 & 1.41 & 1.43 & \\
\hline $\mathrm{H}_{0^{\circ}}$ & & 1.06 & 1.04 & 0.98 & & \\
\hline $\mathrm{L}_{135^{\circ}}$ & & 1.07 & 1.44 & 1.35 & & 1.12 \\
\hline $\mathrm{R}_{45^{\circ}}$ & & 1.07 & 1.30 & 1.21 & & \\
\hline Macaque 3 (anterior) & 102 & & & & & \\
\hline $\mathrm{V}_{90^{\circ}}$ & & 1.44 & 3.36 & 2.33 & 1.47 & \\
\hline $\mathrm{H}_{0^{\circ}}$ & & 1.44 & 2.28 & 1.58 & & \\
\hline $\mathrm{L}_{135^{\circ}}$ & & 1.44 & 2.92 & 2.03 & & 1.03 \\
\hline $\mathrm{R}_{45^{\circ}}$ & & 1.44 & 2.84 & 1.97 & & \\
\hline Macaque 3 (posterior) & 51 & & & & & \\
\hline $\mathrm{V}_{90^{\circ}}$ & & 0.799 & 1.38 & 1.73 & & \\
\hline $\mathrm{H}_{0^{\circ}}$ & & 0.800 & 1.60 & 2.00 & 1.15 & \\
\hline $\mathrm{L}_{135^{\circ}}$ & & 0.789 & 1.60 & 2.03 & & 1.17 \\
\hline $\mathrm{R}_{45^{\circ}}$ & & 0.783 & 1.36 & 1.74 & & \\
\hline Squirrel monkey & 24 & & & & & \\
\hline $\mathrm{V}_{90^{\circ}}$ & & 1.44 & 3.16 & 2.19 & & \\
\hline $\mathrm{H}_{0^{\circ}}$ & & 1.44 & 3.36 & 2.33 & 1.06 & \\
\hline $\mathrm{L}_{135^{\circ}}$ & & 1.44 & 3.44 & 2.38 & & 1.10 \\
\hline $\mathrm{R}_{45^{\circ}}$ & & 1.44 & 3.12 & 2.16 & & \\
\hline Owl monkey & 24 & & & & & \\
\hline $\mathrm{V}_{90^{\circ}}$ & & 0.800 & 2.30 & 2.88 & & \\
\hline $\mathrm{H}_{0^{\circ}}^{\circ}$ & & 0.730 & 1.94 & 2.65 & 1.09 & \\
\hline $\mathrm{L}_{135^{\circ}}$ & & 0.650 & 2.06 & 3.17 & & 1.03 \\
\hline $\mathrm{R}_{45^{\circ}}$ & & 0.780 & 2.54 & 3.26 & & \\
\hline
\end{tabular}

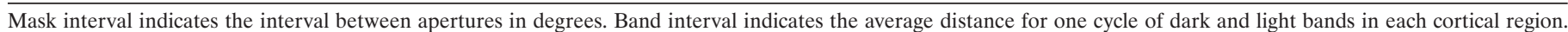

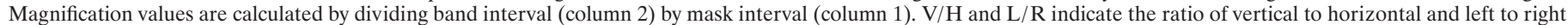
oblique line segments averaged over all quadrangles in each iso-azimuth/iso-elevation grid (Fig. 10b).

plotted the contrast between adjacent light and dark bands as a function of their separation. The graph in Figure $6 e$ shows our results for seven locations, selected from six differential images that we obtained from the same region of cortex in a relatively short period of time. It shows that interband contrast falls linearly with distance, from its highest value determined at the largest separation measured $(4.6 \mathrm{~mm})$ to a zero-intercept at a distance of $1.2 \mathrm{~mm}$. Although measurements at larger intervals might have revealed larger differences, the zero-intercept at $1.2 \mathrm{~mm}$ indicates a lower limit to the scale at which retinotopically resolvable regions can be seen. ${ }^{a}$

\section{Cortical magnification}

From the pronounced difference between the intervals of vertical and horizontal bands in Figures 3, $e$ and $g$, and $4, e$ and $g$, it is clear that magnification is asymmetric and somewhat greater

\footnotetext{
${ }^{a}$ Given the pronounced sensitivity to eye movements, illustrated in Figure 5, it is important to stress that any residual eye movements that occurred during the acquisition of any particular image would reduce the apparent interband contrast, although they would not affect other aspects of these patterns on which our measurements are based. Hence, the observed intercept at $1.2 \mathrm{~mm}$ should be taken as an upper estimate, which may include elements of ocular instability while images were obtained
}

along the axis that represents vertical [an axis that, in this region of cortex, runs perpendicular to ocular dominance columns and parallel to border with V2 (Van Essen et al., 1984; Tootell et al., 1988)]. However, the manner in which these bands have been produced makes it possible to calculate magnification directly by dividing the distance between them (measured in millimeters) by the interval between stimulus apertures. A few examples are summarized in Table 1. From the intervals between horizontal bands in the center of Figure $3 e$ (macaque 1), for example, magnification along the vertical axis appears to be $\approx 3.06 \mathrm{~mm} /{ }^{\circ}$ (millimeters per degree of visual angle). Corresponding parts of Figure $3 g$ (macaque 1 ) indicate a magnification factor of $\approx 2.13$ $\mathrm{mm} /{ }^{\circ}$ along the horizontal axis. Accordingly, the ratio of magnification along vertical and horizontal axes is $\approx 1.4: 1$. Similar calculations applied to corresponding parts of Figure 4 yield vertical and horizontal magnification factors of 2.33 and 1.58 $\mathrm{mm} /{ }^{\circ}$ and a ratio of 1.5:1.

Needless to say, these values agree with previous descriptions that generally reported ratios of $\sim 1.6: 1$ for magnification parallel and perpendicular to the V1/V2 border (Van Essen et al., 1984; Tootell et al., 1988). However, the patterns from which our values are derived reveal more detail that previously has not been available. For example, they show the exact locations and trajec- 

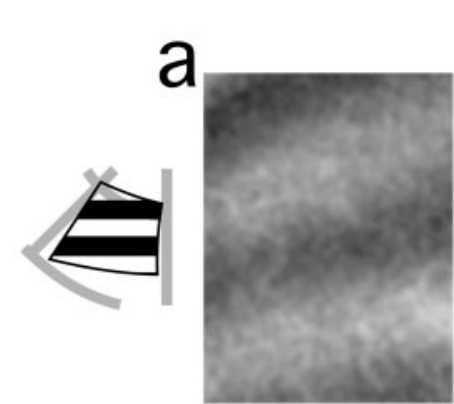

Original

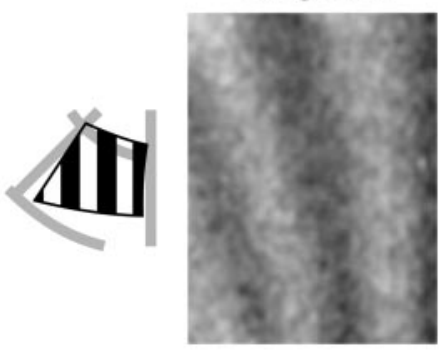

b

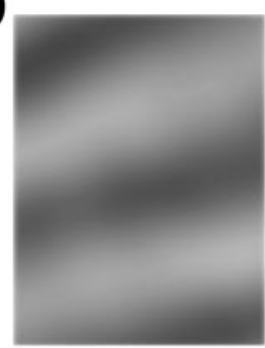

Blur

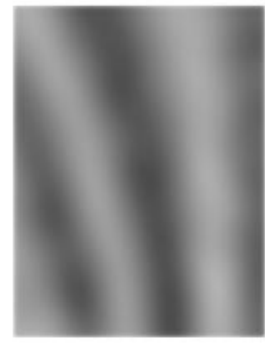

C

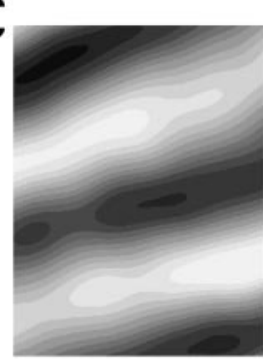

Contour

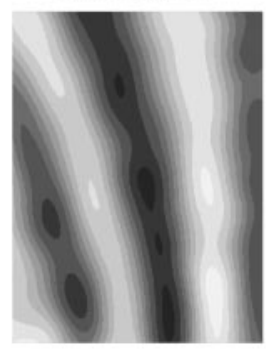

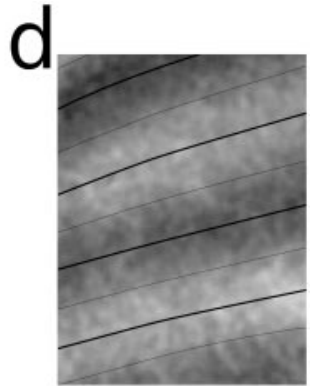

Grid

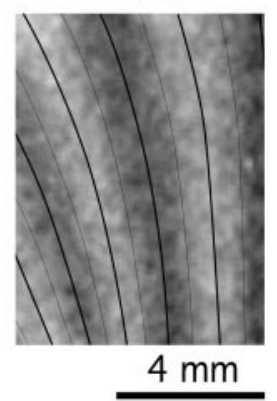

Figure 8. Grid-line representation of the retinotopic patterns induced by vertical $\left(0^{\circ}\right.$; bottom panels) and horizontal $\left(90^{\circ}\right.$; top panels $)$ orientations of repetitive apertures. The pronounced sensitivity of these patterns to lateral displacements (Fig. 5) allows each light/dark cycle to be divided into four phases, represented here by grid lines, which correspond to the centers of adjacent dark and light bands, and the zero-crossings in between. These lines were extracted from a single band pattern, through the following procedure: $a$, we start with the best, artifact-free images of bands representing vertical (bottom panel) and horizontal (top panel) meridia. b. Band-pass filtering (top and bottom panels) removes the high and low frequencies, the periods of which are larger or smaller than the largest and smallest bands in each image. $c$, Contour plots are obtained by reducing the number of gray levels to 16-32 discrete levels and finding edges. Band centers were then isolated from peak values, in the centers of dark and light bands, whereas contours corresponding to zero-crossings were used to delineate the borders. This allows each band cycle to be divided into four, evenly spaced phases, represented by lines, that correspond to the centers of dark and light bands (representing the centers of each set of apertures), and the zero-crossings between them (representing the edges of apertures in visual space). $d$, Superposition of extracted contour lines on the image from $c$. Those in the top frame represent lines of iso-azimuth, whereas those in the bottom frame represent lines of iso-elevation. In subsequent figures, both sets (iso-azimuth and iso-elevation) of lines are combined to form a grid.

tories of ocular dominance columns in each region, and they also reveal the actual representations of equally spaced lines running parallel to horizontal, vertical, and oblique meridia in the visual field. Collectively, these make it possible to answer questions about V1 retinotopy that have not even been posed before.

For example, the patterns in Figures $3, f$ and $g$, and $4, f$ and $g$, make it possible to calculate magnification factors along oblique axes-factors that are important because they can be used to distinguish sources of distortion. If the reported anisotropy in magnification arises only on account of proximity to the V1/V2 border and alignment of the vertical axis with the V1/V2 border, magnification along the two (left and right) oblique axes should be the same. Yet, the values in Table 1 show that they are not: magnification along the left oblique axis exceeds that along the right oblique axis by a slight but consistent amount (e.g., 1.08:1, 1.12:1, and 1.03:1 for macaque 1, 2, and 3). Hence, the observed axis of distortion does not run exactly parallel to the border or the representation of vertical (indicated by the trajectories of bands in Figs. $3 g$ and $4 g$ ). Instead, it appears to tilt counterclockwise by a few degrees.

\section{Iso-azimuth and iso-elevation contours}

Although our procedure for differentially imaging spatial representations does not resolve bands at intervals closer than 1-2 mm, it does contain information about smaller intervals because the patterns of alternating dark and light bands reflect the separate influence of primary and complementary apertures. As a consequence, each dark and light band reflects the influence of a separate aperture, making it possible to represent the activity induced by that aperture with a single line (drawn down its center). Because of the remarkable displacement sensitivity of these patterns (Fig. 5c), additional information can be gleaned from zero-crossings-midpoints between neighboring dark and light bands. Because these represent regions in which responses to both (primary and complementary) sets of apertures were the same, they correspond in principle to regions representing the edges of apertures, and hence can be used to define them. Assuming these assumptions are correct and appropriate, each set of dark and light bands can be used to infer at least four distinct and relatively independent contours: two corresponding to the centers of activity induced by complementary apertures, and two, implied by zero-crossings, corresponding to their common edges.

Figure 8 illustrates our procedure for extracting this information. High-frequency components are first removed (Fig. $8 b$ ) by convolving each image with a Gaussian, whose width has been adjusted to half the width of the smallest visible bands (i.e., one-fourth the period of the narrowest band in any associated image; see Materials and Methods). The values from this result are then binned to infer contours, as illustrated in Figure $8 c$. As one can see, the centers of light and dark bands can be deduced from high and low peaks, whereas midline zero-crossings can be derived from the density of iso-intensity contours in between. Figure $8 d$ shows the derived contours superimposed over the bands used to define them (Fig. $8 a$ ).

The iso-azimuth and iso-elevation lines from Figure $8 d$ define 


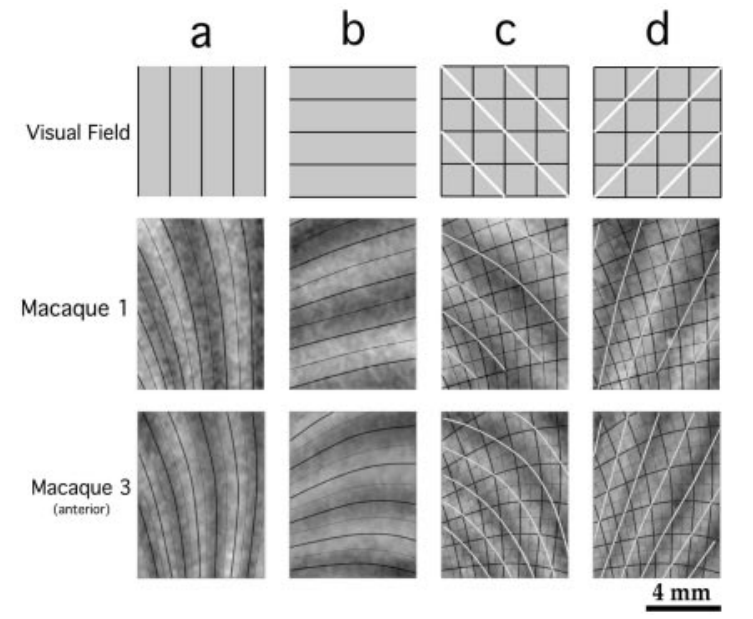

Figure 9. Bands representing oblique apertures run parallel to vertices defined by vertical and horizontal grids. Images in the top row illustrate iso-azimuth $(a)$ and iso-elevation $(b)$ meridia at regular intervals of $1.4^{\circ}$ that are combined in $c$ and $d$ to form square grids. White lines in $c$ and $d$ illustrate how the vertices of these grids connect along left and right oblique trajectories (top row). The second and third rows illustrate results from two animals. In each row, the first two examples show iso-azimuth $(a)$ and iso-elevation $(b)$ contours superimposed over the patterns used to extract them. The third and fourth panels show combined grids superimposed over band patterns that actually were induced by $-45^{\circ}(\mathrm{c})$ and $+45^{\circ}$ $(d)$ apertures. Clearly, the induced bands follow the axes indicated by grid vertices closely. To facilitate comparison, we have connected selected vertices along one of two oblique trajectories with thin white lines. The closeness with which they match emphasizes the internal consistency of these techniques.

a grid of quadrangles (Fig. 10b) that corresponds to a grid of squares (Fig. 10a), defined by equally spaced vertical and horizontal meridia in visual space. Because the vertices of each square connect along $\pm 45^{\circ}$ trajectories, the vertices of quadrangles representing them should connect along axes representing $\pm 45^{\circ}$ as well. Accordingly, it should be possible to test the internal consistency of our assumptions (we made in extracting this information) by comparing the axes defined by grid vertices to the axes of bands that actually were induced by apertures inclined at $\pm 45^{\circ}$. This is done in Figure 9 for two animals, whose images (from Figs. 3 and 4) populate the middle and bottom rows. Diagrams in the top row illustrate corresponding geometries in the visual field.

In the top row, the grid of equally spaced iso-azimuth $(a)$ and iso-elevation $(b)$ contours defines squares ( $c$ and $d$ ) whose vertices connect along trajectories (indicated by thin white lines) of $\pm 45^{\circ}$. The first two images ( $a$ and $b$ ) in the middle and bottom rows show grids of iso-azimuth and iso-elevation contours superimposed over differential images used to derive them. Not surprisingly, they fit. The third and fourth images ( $c$ and $d$ ) show grids derived by superimposing lines from $a$ and $b$, over differential images obtained with apertures inclined at angles of $\pm 45^{\circ}$. As one can see, the dark and light bands induced by left and right oblique apertures run parallel to the thin white lines that have been drawn between grid vertices.

\section{Deformation vectors}

Because each of the extracted quadrangles (Fig. 10b) represents a square in visual space, magnification can also be explored by comparing each one with a $1.4^{\circ}$ by $1.4^{\circ}$ square (Fig. 10a). Although this can be done in a variety of ways, we chose a simple approach that represents each quadrangle with four vectors
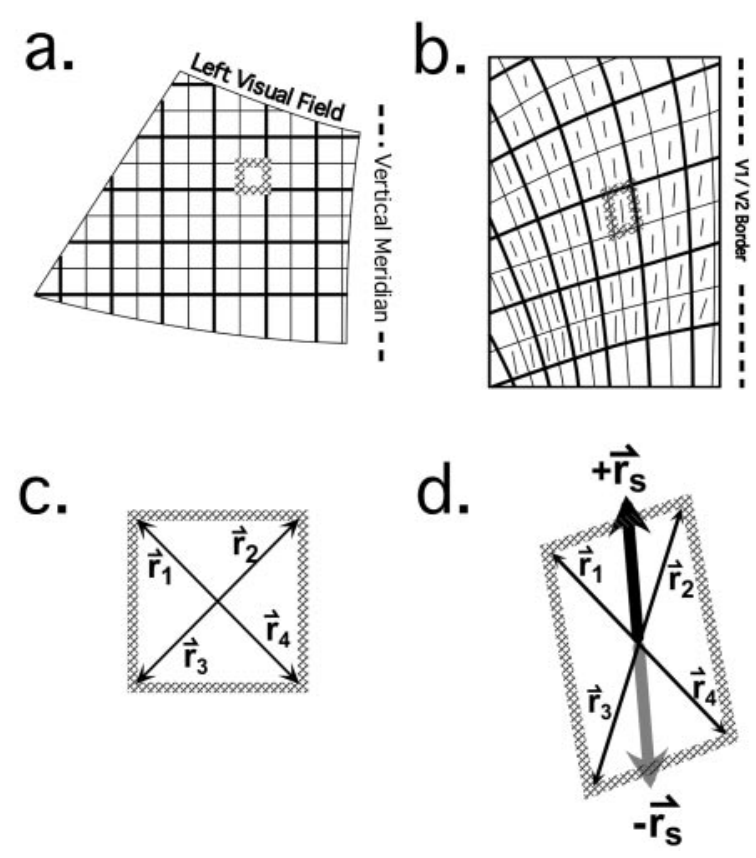

Figure 10. Local measurements of cortical magnification size and symmetry. Because our derived grid (as in Fig. $9 c, d$ ) represents a grid of equally spaced iso-azimuth and iso-elevation meridia $(a)$, each quadrangle in $b$ represents a $0.35 \times 0.35^{\circ}$ square in space. Accordingly, magnification can be quantified for each quadrangle in relation to each square. Although this can be done in several ways, we use a simple approach that entails the calculation of a baseline scalar and the circular variance of vectors used to define each quadrangle. The details are illustrated in $a-d$. As a result, one can calculate magnification and circular variance at each location and represent them with vectors that indicate the direction and degree of distortion. These, in turn, can be compared with ocular dominance patterns directly (Fig. 11). $a$, Visual field projection of the imaged cortex. The unusual shape of its boundary reflects a reversal of the retinocortical distortion applied to the $6.75 \times 9 \mathrm{~mm}^{2}$ rectangle of cortex that we imaged. Note that the numbers of thick and thin lines in $a$ and $b$ are conserved. $b$, This diagram shows the grid we derived from vertical and horizontal band patterns, as explained previously (Fig. 9). From the area and axis of deformation of each quadrangle, it is possible to calculate values for magnification and circular variance at each location. These are indicated by small lines within each quadrangle. The angle and length of each line indicate the axis and degree of deformation. A length of zero indicates no distortion (e.g., a square), whereas a line that touches the edges of the quadrangle indicates infinite distortion in the indicated direction. $c$, Representation of each quadrangle with four normalized vectors. Because the indicated quadrangle in this example is square, in this instance, the circular variance (obtained by squaring each vector and taking the square root of their sum) is zero. $d$, In quadrangles that are not square, however, the resulting vector captures the direction and magnitude of deformation.

whose circular variance (the sum of their squares divided by four times the square of their mean) can be used to calculate a local vector of deformation $\left(\vec{R}_{\mathrm{s}}\right)$ :

$$
\vec{R}_{\mathrm{s}}\left(r_{0}, \omega\right)= \pm \sqrt{\frac{\sum_{i=1}^{4} \vec{r}_{\mathrm{i}}^{2}}{4 \vec{r}_{\mathrm{m}}^{2}}} .
$$

The amplitude $\left(r_{0}\right)$ and angle $(\omega)$ denote the amount and axis of distortion. The ratio of magnification along this axis to magnification along the perpendicular one is given by the relation:

$$
M_{\max } / M_{\min }=\sqrt{\frac{1-r_{0}^{2}}{1+r_{0}^{2}}} .
$$




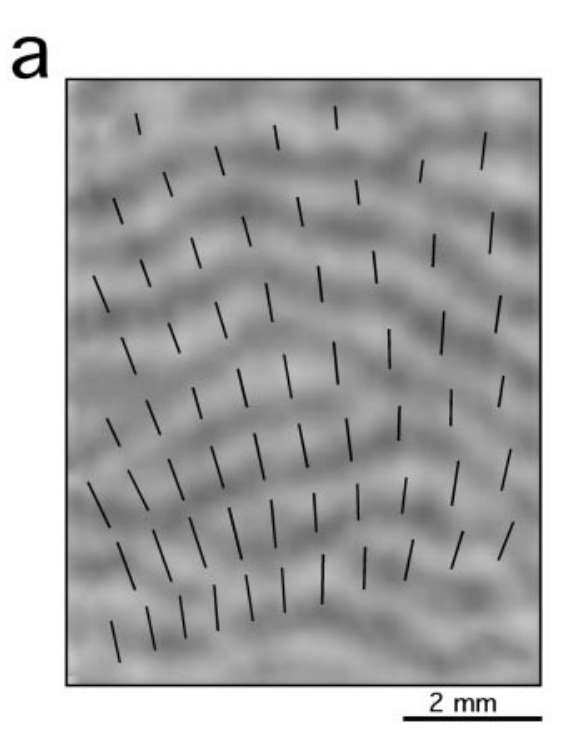

b

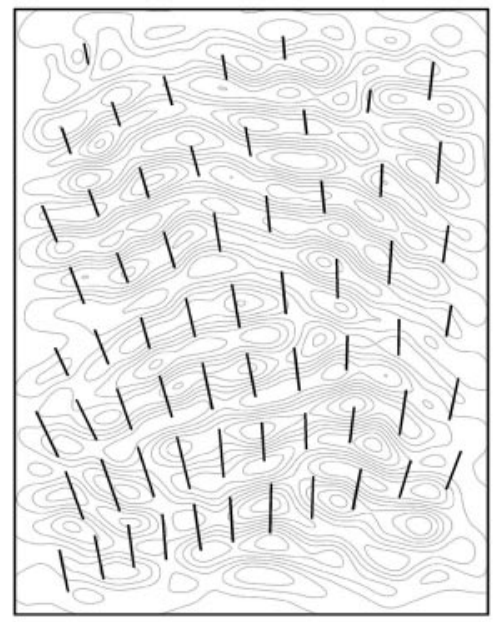

C

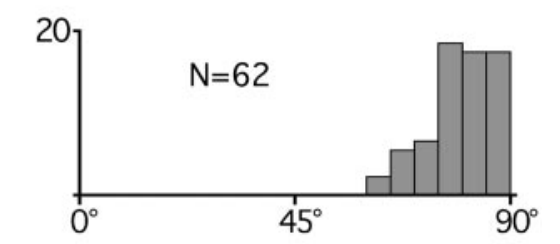

d

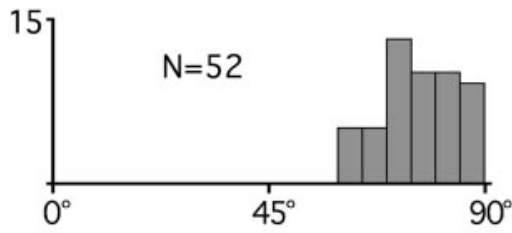

Figure 11. Interaction between ocular dominance columns and local magnification. $a$, This image shows the field of vectors, calculated from the circular variance of quadrangles in Figure 10, superimposed on a differential image of ocular dominance from the same region. The length and angle of each line indicate the degree and axis of distortion; short lines indicate smaller ratios of magnification (approaching 1:1); longer lines indicate greater ratios of magnification along the indicated axes that typically run between 1.4:1 and 1.7:1. As one can predict, most of the ocular dominance columns in this region project parallel to the axis representing horizontal, perpendicular to the V1/V2 border. Hence, the average tendency of superimposed vectors to run parallel to the representation of vertical, along the V1/V2 border, supports previous findings. However, these vectors also provide more detail. By revealing local moments of distortion, in increments of $1 \mathrm{~mm}$, the superimposed lines reveal local ratios of magnification in unprecedented detail, and by revealing them in regions where local patterns of ocular dominance also are known, they make it possible to compare ocular dominance columns with local fluctuations in retinotopy. As one can see, they correspond closely. Even a cursory examination reveals similar trends. For example, there is a gentle clockwise rotation in vector angle that occurs from left to right, matching a corresponding rotation in ocular dominance trajectory. $b$, This image shows the same field of vectors (calculated in Fig. 10) superimposed over an ocular dominance contour plot, derived from iso-intensity values. Whereas ocular dominance trajectories tend to be obvious at large scales, i.e., in regions large enough to contain several repetitions of columns, they are more ambiguous over intervals smaller than single ocular dominance hypercolumns $(\sim 1 \mathrm{~mm})$. However, it is possible to infer them from the gradient of ocular dominance values, which tends to peak at the borders between adjacent columns along axes running perpendicular to ocular dominance column trajectories. Hence, the latter can be estimated from groups of closely spaced contour lines, whose intervals vary inversely with the gradient. By comparing the axis of each vector with that of the nearest group of three or more closely spaced lines, it is possible to estimate angles of intersection, which appear histogrammed in $c$. $c$, This histogram shows the distribution of angles at which vectors intersect ocular dominance columns $a$ and $b$. As one can see, most vectors intersect ocular dominance columns at angles that deviate from perpendicular by $<15^{\circ}$, and none deviate from perpendicular by angles $>30^{\circ}$. $d$, This histogram shows a similar result for 52 vectors obtained from a different animal (macaque 2 in Table 1).

Note the special case in Figure $10 c$, where a quadrangle appears square. In this instance, $r_{0}\left|\vec{R}_{\mathrm{s}}\right| 0$, which implies no distortion, and that $M_{\max } / M_{\min }$ (Eq. 2) is equal to one. For the instance illustrated in Figure $10 d$, on the other hand, where the illustrated quadrangle is far from square, $R_{\mathrm{s}}$ achieves an amplitude of $r_{0}=$ 0.63 , with an inclination of $-8^{\circ}$ (counterclockwise from vertical). This implies that the axis of maximum deformation tilts counterclockwise from vertical by $\sim 8^{\circ}$ and that magnification along it exceeds magnification along its orthogonal by a ratio of $\sim 1.58: 1$ (Eq. 2). In Figure $10 b$ and subsequently, the size and angle of each vector is indicated by a line centered within the quadrangle used to calculate it.

Figure $11 a$ shows lines, representing deformation vectors, superimposed over a differential image of ocular dominance from the same region (Fig. 3b). As one can see, most of these lines intersect ocular dominance bands at angles of $\sim 90^{\circ}$, in agreement with earlier findings (Van Essen et al., 1984; Tootell et al., 1988). They also run vertically, in agreement with the simpler calculations used to derive values in Table 1 and our casual impression that the horizontal bands in Figure $3 g$ appear more widely spaced than the vertical ones in Figure $3 e$.

These vectors contain more information, however, because they reveal actual axes of distortion at frequent intervals. This makes it possible to verify that the general distortion characterizing the entire region arises evenly and continuously from a finer distribution. It does not, for example, reflect as much randomness as one might have guessed from existing estimates of receptive size and scatter. Nor does it indicate pockets of pronounced distortion embedded in a sea that appears more symmetric.

In comparison with the superimposed ocular dominance patterns in Figure 11a, it is clear that the directions and magnitudes of these vectors bear a close relation to the ocular dominance columns in all regions. Even subtle changes in their direction seem to match corresponding shifts in those of neighboring ocular dominance columns. For example, their axes undergo a gentle clockwise rotation from left to right that matches a corresponding trend in ocular dominance trajectory, one that gives it a slightly bowed, upwardly convex appearance. From Figure $11 b$, which shows vectors of distortion superimposed on a map of isodominance contours (derived from Figs. 11a), it is clear that these vectors turn quickly and as often as necessary to maintain steep angles of intersection, angles that, for the most part, lie close to $90^{\circ}$ (Fig. 11c).

The correspondence also extends to vector length and the apparent regularity of ocular dominance columns at each location. As illustrated in Figure 10, the length of each vector indicates the degree of distortion, which can vary from zero (indicating no distortion) to a ratio of $\sim 1.8: 1$ along the indicated axis of the vector. From Figures $11 a$ and $12 d$, however, it is clear that the lengths of these vectors are not random: most vectors appear long 

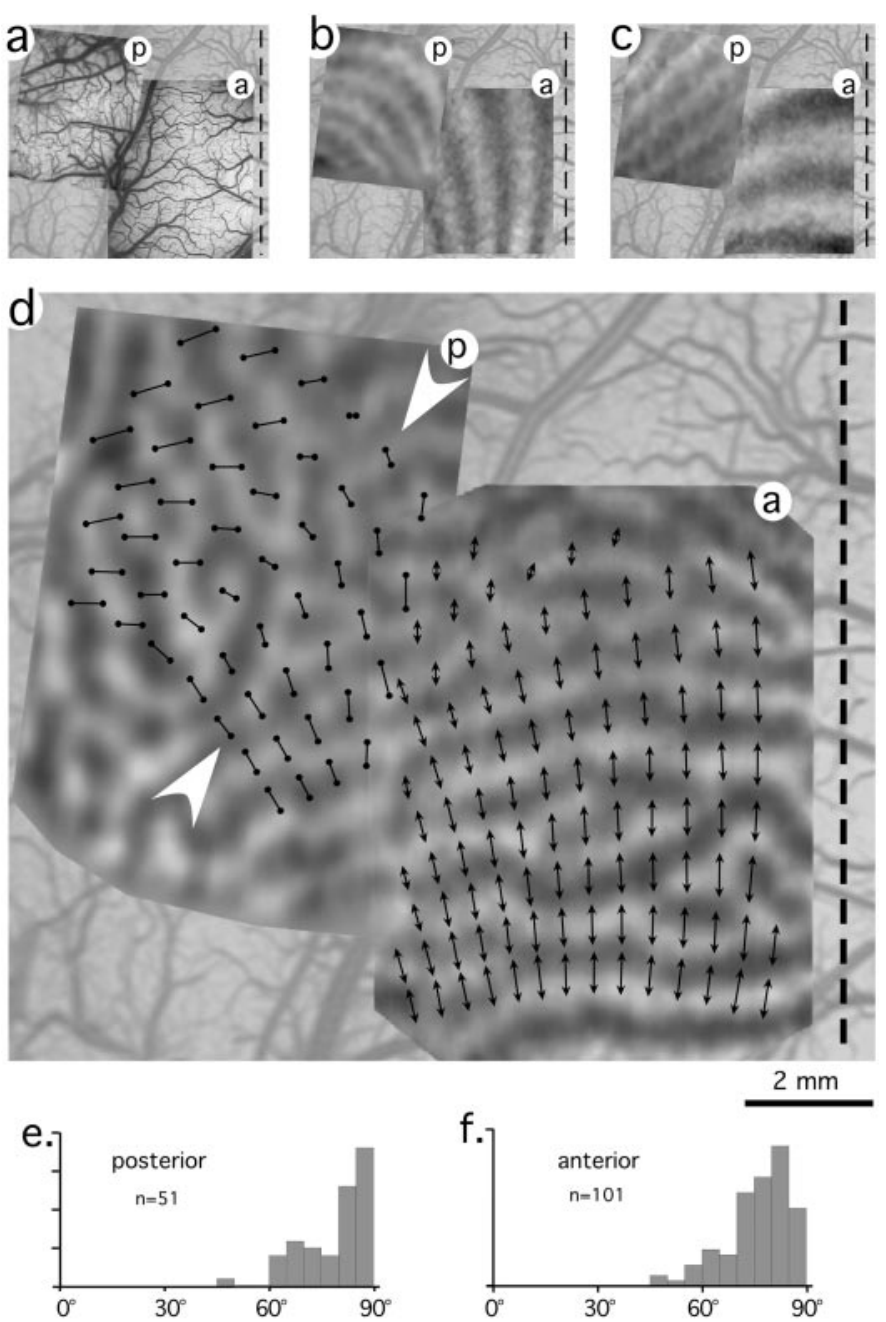

a

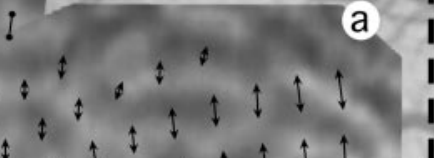

(a)

Figure 12. Interaction between ocular dominance columns and retinotopy over two adjacent regions of cortex in macaque V1. These images combine results from two sequential studies of slightly overlapping regions, one located $6 \mathrm{~mm}$ posterior to the other, in the same animal (macaque 3). As before, all images have been rotated and inverted to bring the V1/V2 border (indicated by broken lines) into alignment with the vertical midline. $a$, Images of the vasculature overlying the two regions of cortex that were explored. These two overlapping images are superimposed on a large-scale, faded image of the surrounding vasculature that we used to align the maps. Note that blood vessels continue across the boundary between these regions. $b$. Patterns of iso-azimuth bands generated in the anterior and posterior regions by vertical apertures. The bands nearest to the V1/V2 border (dashed line) run parallel to it, whereas those further posterior slowly curve away from it, on account of the gradient in magnification. They follow coextensive trajectories at the junction between the anterior and posterior imaged regions, continuing to curve even more until they run perpendicular to the anterior portion of the V1/V2 border. The difference in phase between the anterior and posterior patterns, evident where they join, reflects the different aperture intervals used and the fact that they were obtained at different times (because the eyes or monitor may have moved). Note the contrast with the ocular dominance bands in $d$, where bands imaged separately in the anterior and posterior regions match closely. $c$, Patterns of iso-elevation bands in the anterior and posterior regions of cortex. As in $b$, the bands follow coextensive trajectories, even though they were obtained at different times, and hence with different phases. $d$, Magnification vectors and ocular dominance columns. The anterior and poster images show differential images of ocular dominance that overlap slightly. Dark bands indicate regions dominated by the right eye, whereas light bands indicate regions dominated by the left eye. Superimposed on each of these patterns are distortion vectors, calculated from grids that were extracted from the patterns in $b$ and $c$, according to procedures detailed in Figures 8 and 10. where ocular dominance columns are particularly regular, e.g., near the dorsal V1/V2 border. Shorter ones, if present, tend to lie near instances of sudden trajectory change, e.g., near bifurcations and blind endings along the rift (indicated by arrows) in Figure $12 d$.

Figure 12 shows the same analysis applied to two adjacent regions of cortex that were analyzed in the same animal. As one can see from the scale bar, the posterior region lies $>6 \mathrm{~mm}$ behind the dorsal V1/V2 border on the surface of the operculum, close to the representation of the horizontal meridian, and far enough from the V1/V2 border for columns in the posterior part to pursue a radically different trajectory. From the superimposed vectors, it is clear that local axes of distortion follow suit, changing direction quickly and as often as necessary to remain perpendicular. The precision of coupling and the local directions of distortion suggest that these changes reflect the local influence of ocular dominance columns rather than the V1/V2 border, especially in the posterior region, where ocular dominance columns turn abruptly in the center to run parallel to the dorsal V1/V2 border in the posterior half, and where the accompanying distortion vectors appear to follow suit. As one can see, the short superimposed lines, indicating local axes of distortion, rotate quickly, within a hypercolumn, to stay perpendicular.

The consistently steep angles of intersection (indicated by the histograms in Figs. 11c, 12e,f and Table 2) support a direct link between ocular dominance slabs and local axes of distortion. In fact, the very precision of their alignment with ocular dominance slabs suggests why it may have been difficult to establish this link with previously available techniques. Because of the speed with which ocular dominance columns can change direction, the precision of coupling is likely to produce canceling distortions in neighboring regions, ones that possibly are too close together to be resolved easily by previous techniques. A clear example can be seen in the rift indicated by white arrowheads in Figure $12 d$, on either side of which ocular dominance columns follow dramatically different trajectories. Although our technique reveals an equally dramatic change in the direction of distortion, this would not be apparent at coarser resolutions. If the distortions on either side of this rift were to be combined, for example, they would appear to cancel.

\section{New World primates}

\section{General observations}

The close association between ocular dominance columns and local distortions in Old World primates suggests that species lacking ocular dominance slabs might be characterized by less distortion. To evaluate this hypothesis, we obtained similar maps of retinotopic meridia from two species of New World primate, one squirrel monkey and one owl monkey, neither of which shows

$$
\leftarrow
$$

Vectors calculated for the anterior region end in arrows, whereas vectors calculated for the posterior region end in circles. Note how these vectors cross ocular dominance columns at right angles, turning where they turn to maintain the correlation. The large arrowheads indicate a rift in the posterior region, where ocular dominance bands change trajectory abruptly, from horizontal in the anterior portion to vertical in the posterior one. Note how the distortion vectors keep pace, turning almost as quickly, and how short they become when ocular dominance columns change direction quickly. $e$, The histograms appearing below the large image in $d$ indicate the frequencies of particular angles of intersection, between distortion vectors and ocular dominance columns, in the anterior and posterior regions. As one can see, most intersections occur at angles $>75^{\circ}$, close to $90^{\circ}$. 


\begin{tabular}{|c|c|c|c|c|c|c|c|}
\hline Area & $\mathrm{N}$ & $\mathrm{V} / \mathrm{H}$ & $\mathrm{L} / \mathrm{R}$ & $r^{2}$ & $M_{\max }: M_{\min }$ & $\angle$ & $\mathrm{OD} \angle$ \\
\hline Macaque (anterior) & 102 & 1.47 & 1.03 & 0.37 & $1.47: 1$ & $-3^{\circ}$ & $94^{\circ}$ \\
\hline A & 7 & 1.43 & 1.08 & 0.35 & $1.44: 1$ & $-11^{\circ}$ & $74^{\circ}$ \\
\hline $\mathrm{B}$ & 7 & 1.53 & 1.00 & 0.40 & $1.52: 1$ & $-1^{\circ}$ & $89^{\circ}$ \\
\hline Macaque (posterior) & 51 & 1.15 & 1.17 & 0.11 & $1.12: 1$ & $99^{\circ}$ & $92^{\circ}$ \\
\hline A & 7 & 1.14 & 1.66 & 0.51 & $1.76: 1$ & $75^{\circ}$ & $67^{\circ}$ \\
\hline $\mathrm{B}$ & 7 & 1.24 & 1.18 & 0.27 & $1.31: 1$ & $-19^{\circ}$ & $81^{\circ}$ \\
\hline Squirrel & 24 & 1.06 & 1.10 & 0.09 & $1.09: 1$ & $1^{\circ}$ & - \\
\hline A & 7 & 1.02 & 1.03 & 0.03 & $1.03: 1$ & $6^{\circ}$ & - \\
\hline $\mathrm{B}$ & 7 & 1.07 & 1.10 & 0.12 & 1.13:1 & $7^{\circ}$ & - \\
\hline
\end{tabular}

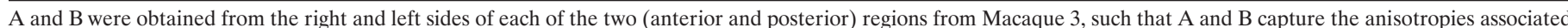

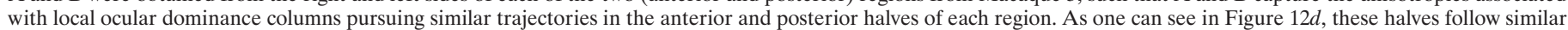

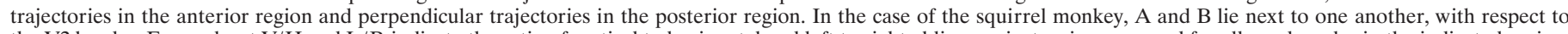

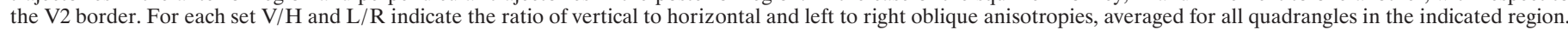

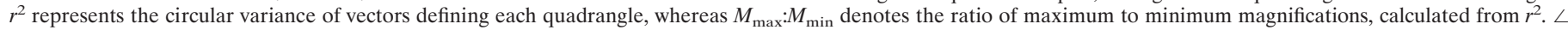
indicates the average orientation with respect to the V1/V2 border, whereas OD $\angle$ reflects the average angle with respect to nearest ocular dominance columns.

pronounced organizations of ocular dominance (Hendrickson et al., 1978; Hubel and Wiesel, 1978).

All the images in Figure 13 were obtained from the same region of squirrel monkey cortex, which has been oriented with the V1/V2 border running vertically, slightly to the right of center. As before, each image has been rotated and inverted to align representations of horizontal and vertical meridia with those of the visual field. The four left-hand images show patterns that have been described briefly (Blasdel et al., 1993), which include: overlying blood vessels $(a)$, differentially imaged responses to vertical and horizontal edges $(b)$, differentially imaged responses to left and right oblique $(c)$, and iso-orientation contours $(d)$ determined from a map of orientation preference (not pictured), that was calculated from eight differential images of orientation selectivity, similar to those in $b$ and $c$.

Images on the right (Fig. 13e-h) show differential responses to stimulus apertures at four orientations (indicated at the right). As for the macaque, the dark and light bands in each pattern reflect the activity induced by stimuli in the primary and secondary sets. Those induced by vertical apertures (Fig. 13e run most parallel to the V1/V2 border and nearly perpendicular to the bands induced by horizontal apertures in Figure $13 g$. The bands induced by left and right oblique apertures (Fig. 13f, h, follow intermediate trajectories. From these and related observations, there is little doubt that each set of bands reflects activity induced by a particular set of apertures in the visual field. Although we did not investigate the resolution of retinotopic maps in New World primates directly, one can see interactions that reveal something about the coarser resolution of V2 where neurons, because of their larger receptive fields, did not distinguish strongly between stimuli in our primary and complementary apertures. Accordingly, the induced bands fade abruptly at the V1/V2 border.

Considering that the bands in each image (Fig. 13e-h) reflect the spatial geometries of apertures used to make them (indicated to the right of each image), it is surprising that their alignment with the V1/V2 border does not reflect that between the inducing apertures and the vertical meridian. In contrast to macaques (Figs. $3 e, g 4 e, g$ ), where the bands induced by vertical and horizontal apertures run parallel and perpendicular to the V1/V2 border, the axes represented by vertical and horizontal bands in Figure 13, $e$ and $g$, appear offset by angles of $\sim 20^{\circ}$ and $30^{\circ}$ (Fig. 14e). This tilt does not result from cyclo-torsion (e.g., associated with paralysis), because the trajectories of bands produced sepa- rately through either eye are the same. Because it is too large to be explained by chance or individual variation, it appears that the axis represented along the V1/V2 border in each of these two species of New World primate tilts with respect to vertical by $\sim 20-30^{\circ}$.

\section{New World magnification}

Because the bands in Figure $13 e-h$ were induced by apertures repeating at the same interval, the similar spacing of those induced by vertical and horizontal apertures in Figure 13, $e$ and $g$, indicates little or no anisotropy in magnification. This becomes particularly evident from the grid of iso-azimuth and isoelevation contours in Figure 14e. As one can see, the resulting quadrangles appear more symmetric than those obtained from macaques (Fig. 10b). Although they still reflect some elongation parallel to the V1/V2 border (particularly evident from their diamond-shapes and from the short but finite vectors within them), the indicated anisotropy (1.09:1) is still seven times smaller than that calculated (on the basis of similar measurements) for macaques. The 1.07:1 ratio obtained for the same part of cortex from the owl monkey (Table 2) was smaller still. In addition to correlating with the axes of ocular dominance columns in Old World primates, therefore, the presence of pronounced distortions seems to depend on them.

Because magnification does not appear to be exactly isotropic in New World primates (i.e., the ratio is 1.09:1 rather than 1:1), it is tempting to attribute the minor discrepancy to some other factor, for example, to some residual expression of ocular dominance in these species. Although trace ocular dominance organizations have been described, however, they seem unlikely to explain this distortion, because most of those described appear as patches, rather than slabs, with no obvious alignment to the V1/V2 border (Horton and Hocking 1996; Livingstone, 1996).

Iso-orientation slabs offer another possibility, however, because narrow clusters of them do appear to intersect the V1/V2 border at right angles in squirrel monkeys (Fig. 13b-d), owl monkeys (Sincich, 1999), and a number of other species that lack pronounced organizations of ocular dominance slabs (Humphrey et al., 1980; Bosking et al., 1997). Accordingly, the small anisotropies in Figure 14e might reflect the influence of iso-orientation slabs, whose multiple representations of different orientations for the same parts of space might also induce distortions. To evaluate this possibility, we compared the vectors in Figure 14e with one 

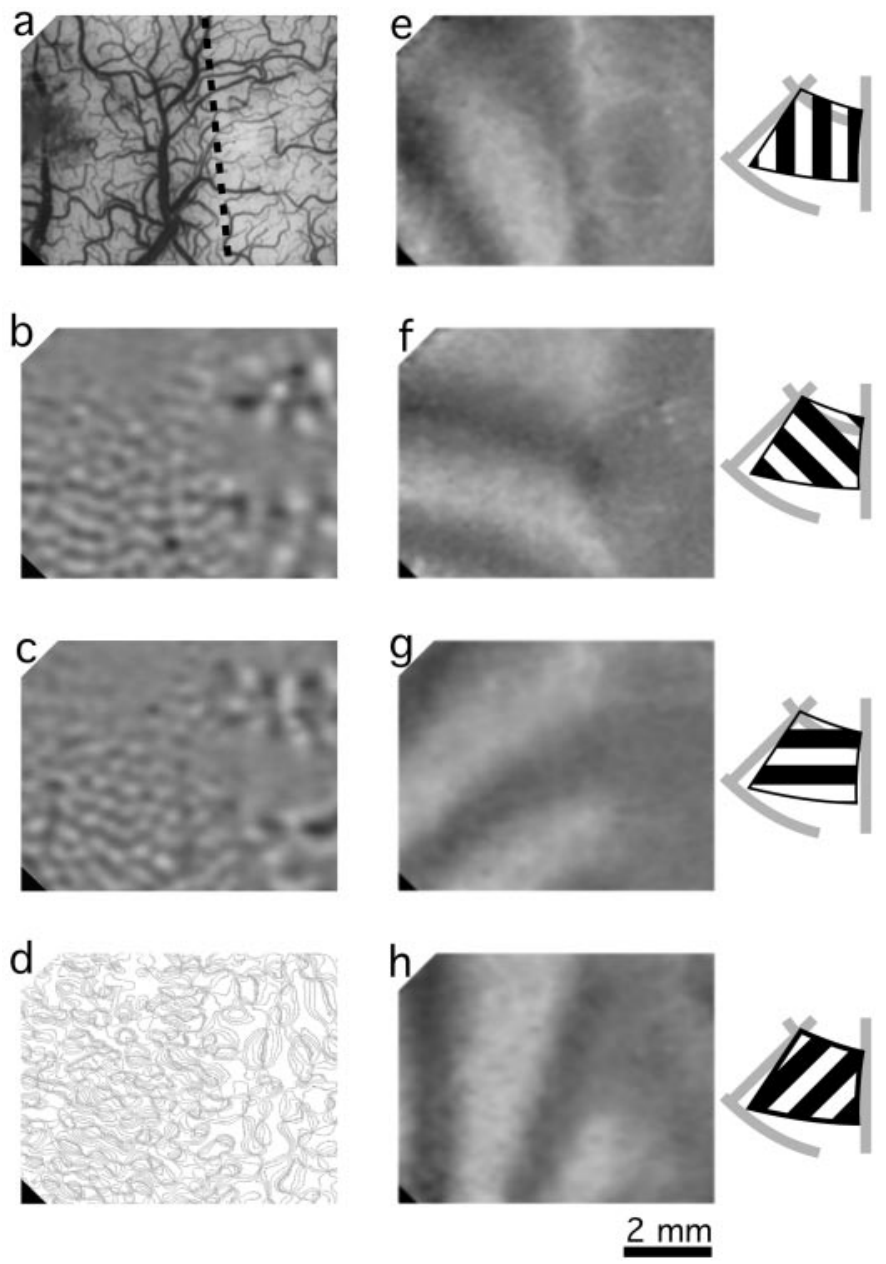

Figure 13. Functional maps of orientation selectivity and retinotopic meridia in V1 and V2 of the squirrel monkey. Each of these images was obtained from the same region of cortex, centered over corresponding regions of $\mathrm{V} 1$ and $\mathrm{V} 2$, with the border between them oriented vertically through the center. $a$, Overlying vasculature. In the squirrel monkey, the density of vascularization frequently heralds the transition between V1 and V2. This clearly is evident in this image, which shows a darker V1 on the left. $b$, Pattern of orientation preferences for vertical and horizontal. Because this image was obtained by subtracting cortical responses to horizontal stimuli from cortical responses to vertical stimuli, dark and light regions indicate preferences for vertical and horizontal. Note a clear transition at the V1/V2 border: on the left, in V1, a fine pattern of dark and light iso-orientation bands run perpendicular to the V1/V2 border; on the right, in V2, a much coarser organization of orientation selectivity patterns appears to radiate outward, to the right, in thick bands that correlate in location with thick cytochrome oxidase bands (Blasdel et al., 1993; Horton et al., 1996). c, Orientation pattern produced by left and right oblique stimuli. Dark and light zones indicate preference for left and right oblique. $d$, Iso-orientation contours calculated from eight differential images of orientation (including those in $b$ and $c$ ). The horizontal trajectories of most lines on the left side of the V1/V2 border confirm the results from $b$ and $c$, that contours of iso-orientation intersect the V1/V2 border at angles of $\sim 90^{\circ}$. $e-h$, Patterns of retinotopic position elicited by apertures, $0.7^{\circ}$ wide repeated at intervals of $1.4^{\circ}$, and oriented vertically $(e)$, along the left oblique $(f)$, horizontally $(g)$, and along the right oblique $(h)$. The bands in each pattern terminate at the V1/V2 border and all appear to be offset (by $20-30^{\circ}$ ) from axes defined with respect to the V1/V2 border.

differential image of orientation in Figure 14f. As one can see, the small deformation vectors cross iso-orientation contours at steep angles (Fig. $14 g$ ) that always exceed $45^{\circ}$. And although more samples clearly are needed, some correlation seems evident from the histogram in Figure $14 g$.

\section{DISCUSSION}

These results demonstrate a new procedure for mapping cortical retinotopy in vivo, one that makes it possible to visualize representations of specific meridia with unprecedented resolution. By comparing these patterns with stimuli used to make them, it is possible to infer many aspects of transforms in between. One concerns the resolution with which specific parts of primary visual cortex represent specific parts of space, another concerns magnification (the relative areas of cortex and represented parts of space), and yet another concerns interaction with other response properties that are organized laterally in V1.

\section{Retinotopic resolution}

Before addressing the scale and symmetry of magnification, it is important to consider the resolution of V1, the relative separation of regions representing nonoverlapping parts of the visual field. Because of the ease with which our technique resolves ocular dominance and orientation patterns (Figs. $3 b-d, 4 b-d$ ), there is little doubt that it easily can resolve features finer than $300 \mu \mathrm{m}$. Still, there is no hint of retinotopically induced bands lying closer than $1.2 \mathrm{~mm}$, and even at larger intervals the contrast is impaired over intervals $<4-5 \mathrm{~mm}$. This suggests a lower limit to the retinotopic representation of visual space in V1.

One caveat to this observation concerns the possibility that eye movements may have obscured this measure; any residual eye movements during imaging would have increased our estimate of this limit by attenuating contrast and shifting the regression line rightward in Figure 6e. Movements during paralysis rarely exceed $0.1^{\circ}$, however, and those that do occur are usually transient (Blasdel and Fitzpatrick, 1984). Nevertheless, a worst case scenario, in which $0.1^{\circ}$ movements occur regularly enough to obscure resolution, would account for only $\approx 0.3 \mathrm{~mm}$ at this eccentricity (Fig. 5), leaving a $900 \mu \mathrm{m}$ gap within which signals associated with two alternately activated sites are difficult or impossible to distinguish. This would imply that regions activated by stimuli in each aperture have central plateaus that are at least $0.9-1.2 \mathrm{~mm}$ wide. This dimension agrees surprisingly well with that of single magnocellular afferents that have been observed to spread $\sim 1$ $\mathrm{mm}$ in layer $4 \mathrm{c} \alpha$ (Blasdel and Lund, 1983). The linear increase in contrast with intervals $>1.2 \mathrm{~mm}$ similarly might be explained by the tapering overlap of regions excited by patchy lateral projections that reportedly spread laterally for 2-3 $\mathrm{mm}$ (Rockland and Lund, 1983; Lund and Yoshioka, 1991; Malach et al., 1993; Lund et al., 1994; Weliky et al., 1995; Yoshioka et al., 1996; Sincich, 1999). Within a particular animal, these measures seem likely to provide a precise and repeatable measure of retinotopic resolution. In view of the variability that characterizes ocular dominance slabs in macaque monkeys (Horton and Hocking, 1996a), it will be interesting to see whether this measurement of resolution varies as well, whether it is strongly conserved in different animals or changes with the scale of ocular dominance and orientation columns.

\section{Magnification}

Although it is not possible to image retinotopic bands at intervals closer than a few millimeters, the remarkable displacement sensitivity (illustrated in Fig. 5) indicates that elements of retinotopic precision are retained. Even though fine lines in space cannot be represented by fine lines in cortex, intervals between them can. 

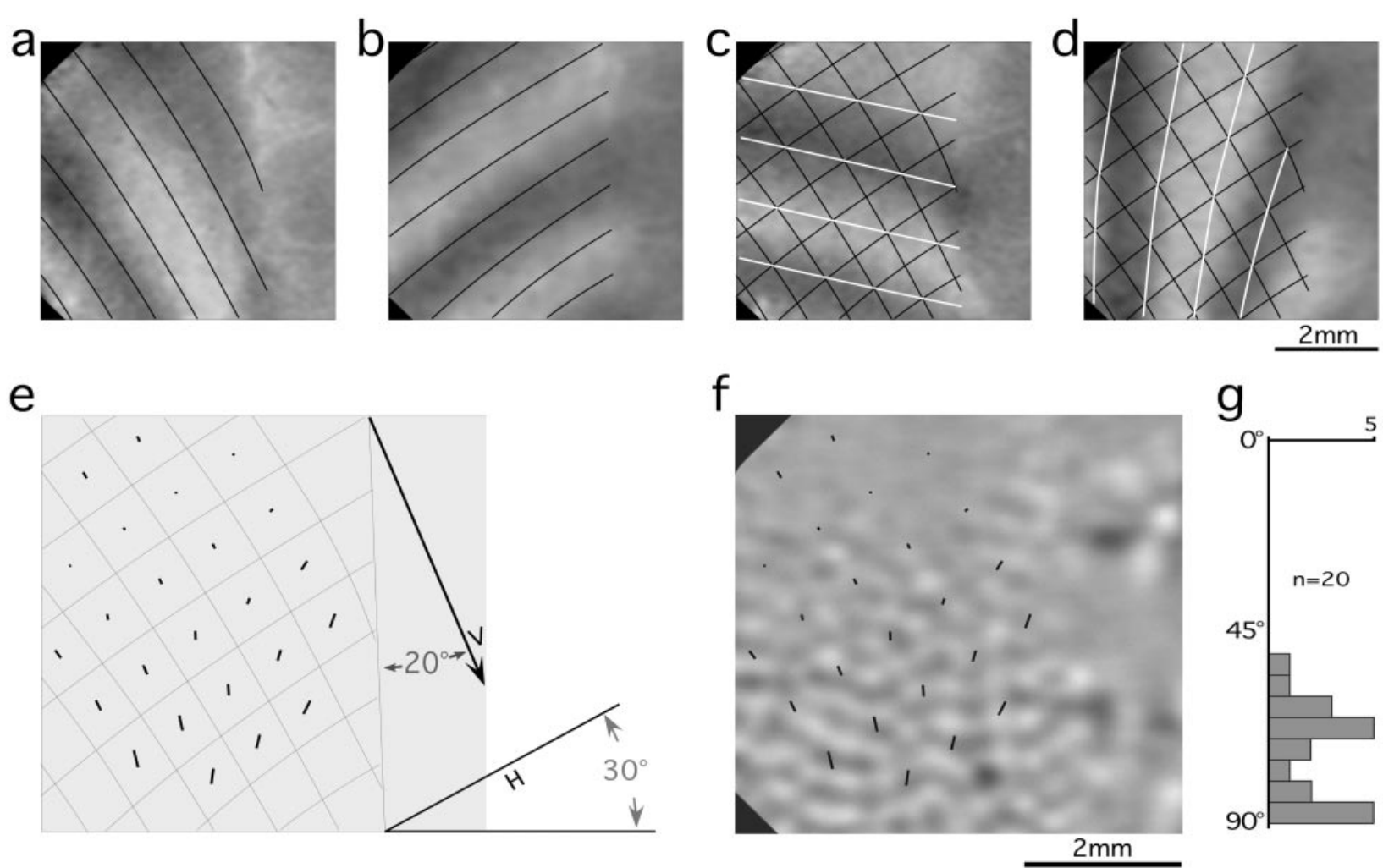

Figure 14. Patterns of orientation and magnification in the squirrel monkey. $a$, Iso-azimuth contours superimposed over the bands induced by vertical apertures, from which we extracted them. $b$, Iso-elevation contours superimposed over bands induced by horizontal apertures. $c$, Superposition of the grid, constructed from iso-azimuth and iso-elevation contours $(a, b)$, with the differential image of bands induced by apertures inclined at $+45^{\circ}$ (left oblique). As one can see, these bands run parallel to the thin white lines that connect selected vertices at left oblique angles. $d$, Superposition of the same grid with the differential image of bands induced by $135^{\circ}$ apertures. As one can see, both the light and dark bands follow the same trajectories as the fine white lines that connect selected vertices at right oblique angles. $e$, This diagram shows the grid of iso-azimuth and iso-elevation contours up to the V1/V2 border (indicated by vertical line) and the vectors calculated from the circular variance of the quadrangle vertices defined by each quadrangle. As one can see, these vectors still run parallel to the V1/V2 border, although they clearly are much smaller than those obtained from macaque V1 (Figs. 11, 12). Note also how the axes of iso-azimuth and iso-elevation contours, which should run vertical and horizontal, deviate by $20-30^{\circ}$ from the axes defined with respect to the V1/V2 border. $f$, Superposition of the distortion vectors, calculated from the circular variance of each quadrangle, over one differential image of orientation preference obtained from Figure $13 \mathrm{~b}$. These clearly run perpendicular to the dark and light bands that indicate contours of iso-orientation. $g$, This histogram shows the distribution of angles at which local distortion vectors intersect axes of iso-orientation at 20 different locations. Many occur at angles approaching $90^{\circ}$. All intersect at angles steeper than $45^{\circ}$.

Accordingly, intervals between the iso-azimuth and the isoelevation contours in Figure $10 b$ can accurately represent the intervals between iso-azimuth and iso-elevation meridia in space (Fig. 10a), and they can be used to calculate the scale and symmetry of magnification.

At the coarsest level of analysis, the different intervals between vertical and horizontal bands in Figure 3, $e$ and $g$ (as well as Fig. $4, e$ and $g$ ) may be used to infer greater magnification along the vertical axis which, for this part of cortex, runs parallel to the V1/V2 border (Van Essen et al., 1984; Tootell et al., 1988). These patterns also make it possible to calculate magnification vectors that reveal the local symmetry of magnification at the scale of ocular dominance (and orientation) columns. As one can see for two different animals in Figures 11, $a$ and $b$, and $12 d$, these show the same general distortion, parallel to the V1/V2 border. However, they also reveal local variations that seem to match corresponding changes in ocular dominance trajectories. Accordingly, they provide an additional correlation at the scale of ocular dominance columns, which is finer than any shown before.

Because this technique can be used in any accessible region of cortex, it can be used to analyze these relations in regions that have proved difficult to analyze before. From the distortions we found in the posterior part of Figure $12 b$ (region p), far from the V1/V2 border, it is clear that local distortions in retinotopy are the rule rather than the exception and that the observed directions of distortion run perpendicular to ocular dominance columns. Accordingly, we conclude: (1) that ocular dominance columns (or factors common to ocular dominance and retinotopy) drive this distortion, and (2) that the main reason that magnification appears amplified along the V1/V2 border is that ocular dominance columns tend to intersect the border at right angles (LeVay et al., 1975).

\section{New World primates}

To test the relationship between ocular dominance and distortion further, we obtained similar patterns from the primary visual cortex of two species of New World primate that are known to lack pronounced, slab-like, ocular dominance organizations. In contrast to macaques, in which we regularly observed magnification ratios of 1.6:1, maps from the primary visual cortex of one squirrel (Figs. 13, 14) and one owl monkey (Table 2) showed ratios of only 1.09:1 and 1.07:1. Accordingly, they support a link between distortion and slab-like ocular dominance columns.

Although it is small, there is a finite, retinotopic distortion in New World primates that could reflect other organizations. If so, 
the possibility that it reflects residual ocular dominance organizations [e.g., those observed with single-unit recordings (Hubel and Wiesel, 1974b], seems unlikely because (1) techniques that show ocular dominance columns easily in Old World primates do not find them in these species, and (2) patterns of ocular dominance that have been seen in New World primates (Horton and Hocking 1996b; Livingstone, 1996) appear patchy rather than banded, with little obvious alignment to the V1/V2 border. Hence, even if they distort retinotopy, it is not clear why they would do so along the V1/V2 border.

Because iso-orientation slabs intersect the V1/V2 border at right angles in New World primates (Blasdel et al., 1993) (Fig. $13 b-d)$, they correlate directly with the observed direction of distortion. Although a relationship remains to be established, this correlation does suggest a general principle: that organizations of any response property in linear arrays of slabs might generally distort space by increasing magnification (through the replication of regions representing the same parts of space, in adjacent slabs) in one dimension. If so, the smaller anisotropy ( 9 vs $60 \%$ ) observed in squirrel monkey V1 might reflect the dimensions of orientation slabs, which tend to appear more narrow and less regular than ocular dominance slabs in macaques. In view of the known orthogonality between orientation and ocular dominance slabs in macaque monkeys (Obermayer and Blasdel, 1993), any tendency by orientation slabs to increase magnification perpendicular to their axes could explain another discrepancy in macaque V1: why the distortions associated with ocular dominance columns always fall short of the 2:1 ratio that would be expected for a duplication of space in flanking (ocular dominance) columns.

\section{REFERENCES}

Allman JM, Kaas JH (1971) Representations of the visual field in striate and adjoining cortex of the owl monkey (Aotus trivirgatus). Brain Res 35:89-106.

Blasdel GG (1992a) Differential imaging of ocular dominance and orientation selectivity in monkey striate cortex. J Neurosci 12:3115-3138.

Blasdel GG (1992b) Orientation selectivity, preference, and continuity in monkey striate cortex. J Neurosci 12:3141-3163.

Blasdel GG, Fitzpatrick D (1984) Physiological organization of layer 4 in macaque striate cortex. J Neurosci 4:880-895.

Blasdel GG, Lund JS (1983) Termination of afferent axons in macaque striate cortex. J Neurosci 3:1389-1413.

Blasdel GG, Salama G (1986) Voltage-sensitive dyes reveals a modular organization in the monkey striate cortex. Nature 321:579-585.

Blasdel GG, Livingstone MS, Hubel DH (1993) Optical imaging of orientation and binocularity in visual areas 1 and 2 of squirrel monkey (Samiri sciureus) cortex. Soc Neurosci Abstr 19:618.5.

Bonhoeffer T, Grinvald A (1993) The layout of iso-orientation domains in area 18 of cat visual cortex: optical imaging reveals a pinwheel-like orientation. J Neurosci 13:4157-4180.

Bosking WH, Zang Y, Schofield B, Fitzpatrick D (1997) Orientation selectivity and the arrangement of horizontal connections in tree shrew striate cortex. J Neurosci 17:2212-2227.

Bressloff PC, Cowan JD, Golubitsky M, Thomas PJ, Wiener MC (2001) Geometric visual hallucinations, Euclidean symmetry and the functional architecture of striate cortex. Philos Trans R Soc Lond B Biol Sci 356:299-330

Daniel PM, Whitteridge D (1961) The representation of the visual field on the cerebral cortex in monkeys. J Physiol (Lond) 159:203-221.

Grinvald A, Lieke E, Frostig RP, Gilbert C, Wiesel RM (1986) Functional architecture of cortex revealed by optical imaging of intrinsic signals. Nature 324:351-364.

Hendrickson AE, Wilson JR, Ogren MP (1978) The neuroanatomical organization of pathways between the dorsal lateral geniculate nucleus and visual cortex in Old World and New World primates. J Comp Neurol 182:123-136.

Horton JC, Hocking DR (1996a) Intrinsic variability of ocular domi- nance column periodicity in normal macaque monkeys. J Neurosci 16:7228-7339.

Horton JC, Hocking DR (1996b) Anatomical demonstration of ocular dominance columns in striate cortex of the squirrel monkey. J Neurosci 17:5510-5522.

Hubel DH, Freeman DC (1977) Projection into the visual field of ocular dominance columns in macaque monkey. Brain Res 122:336-343.

Hubel DH, Wiesel TN (1962) Receptive fields binocular interaction and functional architecture of monkey striate cortex. J Physiol (Lond) 160:106-154.

Hubel DH, Wiesel TN (1974a) Sequence regularity and geometry of orientation columns in the monkey striate cortex. J Comp Neurol 158:267-294.

Hubel DH, Wiesel TN (1974b) Uniformity of monkey striate cortex: a parallel relationship between field size, scatter and magnification factor. J Comp Neurol 158:295-306.

Hubel DH, Wiesel TN (1977) Functional architecture of macaque visual cortex. Ferrier lecture. Proc R Soc Lond B Biol Sci 198:1-59.

Hubel DH, Wiesel TN (1978) Distribution of inputs from the two eyes to striate cortex of squirrel monkeys. Soc Neurosci Abstr 4:2018.

Hubel DH, Wiesel TN, LeVay S (1974) Visual field of representation in layer IVC of monkey striate cortex. Soc Neurosci Abstr 10:264.

Humphrey AL, Skeen LC, Norton TT (1980) Topographic organization of the orientation column system in the striate cortex of the tree shrew. J Comp Neurol 192:549-566.

LeVay S, Hubel DH, Wiesel TN (1975) The pattern of ocular dominance columns in macaque visual cortex revealed by reduced silver stain. J Comp Neurol 159:559-576.

Livingstone MS (1996) Ocular dominance columns in New World monkeys. J Neurosci 16:2086-2096.

Lund JS, Yoshioka T (1991) Local circuit neurons of macaque monkey striate cortex: IV Neurons of laminae 1-3A. J Comp Neurol 311:234-259.

Lund JS, Yoshioka T, Levitt JB (1994) Substrates for interlaminar connections in area V1 of macaque monkey cerebral cortex. In: Cerebral cortex (Peters A and Rockland KS, eds), pp 37-60. New York: Plenum.

Malach, R, Amir Y, Harel M, Grinvald A (1993) Relationship between intrinsic connections and functional architecture revealed by optical imaging and in vivo targeted biocytin injections in primate striate cortex. Proc Natl Acad Sci USA 90:10469-10473.

McLoughlin NP, Blasdel GG (1998) Wavelength-dependent differences between optically determined functional maps from the macaque striate cortex. NeuroImage 7:326-336.

Obermayer K, Blasdel GG (1993) Geometry of orientation and ocular dominance columns in monkey striate cortex. J Neurosci 13:4114-4129.

Penfield W (1933) The evidence for a cerebral vascular mechanism in epilepsy. Ann Intern Med 7:303-310.

Rockland KS, Lund JS (1983) Intrinsic laminar lattice connections in primate visual cortex. J Comp Neurol 216:303-318.

Sakitt B (1982) Why the cortical magnification factor in Rhesus can not be isotropic. Vision Res 22:417-421.

Schwartz EL (1977) Spatial mapping in primate sensory projection: analytic structure and relevance to perception. Biol Cybern 25:181-194.

Schwartz EL (1985) On the mathematical structure of the retinotopic mapping of primate striate cortex. Science 227:1066.

Sincich L (1999) Local circuits in the primary visual cortex. PhD thesis, Harvard University.

Tootell RB, Silverman MS, Switkes E, De Valois RL (1982) Deoxyglucose analysis of retinotopic organization in primate striate cortex. Science 218:902-904.

Tootell RB, Switkes E, Silverman MS, Hamilton SL (1988) Functional anatomy of macaque striate cortex. II. Retinotopic organization. J Neurosci 8:1531-1568.

Ts'o DY, Frostig RD, Lieke EE, Grinvald A (1990) Functional organization of primate visual cortex revealed by high resolution optical imaging. Science 249:417-420.

Van Essen DC, Newsome WT, Maunsell JH (1984) The visual field representation in striate cortex of the macaque monkey: asymmetries, anisotropies, and individual variability. Vision Res: 24:429-448.

Weliky M, Kandler K, Fitzpatrick D, Katz LC (1995) Patterns of excitation and inhibition evoked by horizontal connections in visual cortex share a common relationship to orientation columns. Neuron 15:541-552.

Wolbarsht ML, MacNichol EF, Wagner HG (1960) Glass insulated platinum microelectrode. Science 132:1309-1310.

Yoshioka T, Blasdel GG, Levitt JB, Lund JS (1996) Relation between patterns of intrinsic lateral connectivity, ocular dominance, and cytochrome oxidase-reactive regions in macaque monkey striate cortex. Cereb Cortex 6:297-310. 